\title{
Multimodality carotid plaque tissue characterization and classification in the artificial intelligence paradigm: a narrative review for stroke application
}

\author{
Luca Saba ${ }^{1}$, Skandha S. Sanagala ${ }^{2,3} \wedge$, Suneet K. Gupta ${ }^{3} \wedge$, Vijaya K. Koppula ${ }^{2} \wedge$, Amer M. Johri ${ }^{4} \wedge$, \\ Narendra N. Khanna ${ }^{5} \wedge$, Sophie Mavrogeni ${ }^{6} \wedge$, John R. Laird ${ }^{7} \wedge$, Gyan Pareek ${ }^{8} \wedge$, Martin Miner ${ }^{9} \wedge$, \\ Petros P. Sfikakis ${ }^{10} \wedge$, Athanasios Protogerou ${ }^{11} \wedge$, Durga P. Misra ${ }^{12}$, Vikas Agarwal $^{12} \wedge$, Aditya M. Sharma ${ }^{13} \wedge$, \\ Vijay Viswanathan $^{14} \wedge$, Vijay S. Rathore ${ }^{15} \wedge$, Monika Turk ${ }^{16}$, Raghu Kolluri ${ }^{17} \wedge$, Klaudija Viskovic ${ }^{18} \wedge$, \\ Elisa Cuadrado-Godia ${ }^{19} \wedge$, George D. Kitas ${ }^{20}$, Neeraj Sharma ${ }^{21}$, Andrew Nicolaides ${ }^{22}$, Jasjit S. Suri ${ }^{23}$
}

${ }^{1}$ Department of Radiology, Azienda Ospedaliero Universitaria (AOU), Cagliari, Italy; ${ }^{2}$ CSE Department, CMR College of Engineering \& Technology, Hyderabad, India; ${ }^{3}$ CSE Department, Bennett University, Greater Noida, UP, India; ${ }^{4}$ Department of Medicine, Division of Cardiology, Queen's University, Kingston, Ontario, Canada; ${ }^{5}$ Department of Cardiology, Indraprastha APOLLO Hospitals, New Delhi, India; ${ }^{6}$ Cardiology Clinic, Onassis Cardiac Surgery Center, Athens, Greece; ${ }^{7}$ Heart and Vascular Institute, Adventist Health St. Helena, St Helena, CA, USA; ${ }^{8}$ Minimally Invasive Urology Institute, Brown University, Providence, Rhode Island, USA; ${ }^{9}$ Men's Health Center, Miriam Hospital Providence, Rhode Island, USA; ${ }^{10}$ Rheumatology Unit, National Kapodistrian University of Athens, Greece; ${ }^{11}$ Department of Cardiovascular Prevention, National and Kapodistrian University of Athens, Athens, Greece; ${ }^{12}$ Department of Clinical Immunology and Rheumatology, SGPGIMS, Lucknow, India; ${ }^{13}$ Division of Cardiovascular Medicine, University of Virginia, VA, USA; ${ }^{14}$ MV Hospital for Diabetes \& Professor M Viswanathan Diabetes Research Centre, Chennai, India; ${ }^{15}$ Nephrology Department, Kaiser Permanente, Sacramento, CA, USA; ${ }^{16}$ The Hanse-Wissenschaftskolleg Institute for Advanced Study, Delmenhorst, Germany; ${ }^{17}$ OhioHealth Heart and Vascular, OH, USA; ${ }^{18}$ University Hospital for Infectious Diseases, Zagreb, Croatia; ${ }^{19}$ IMIM - Hospital Del Mar, Passeig Marítim, Barcelona, Spain; ${ }^{20} \mathrm{R} \&$ D Academic Affairs, Dudley Group NHS Foundation Trust, Dudley, UK; ${ }^{21}$ Department of Biomedical Engineering, IIT-BHU, Banaras, UP, India; ${ }^{22}$ Vascular Screening and Diagnostic Centre, University of Nicosia, Nicosia, Cyprus; ${ }^{23}$ Stroke Monitoring and Diagnostic Division, AtheroPoint ${ }^{\mathrm{TM}}$, Roseville, CA, USA

Contributions: (I) Conception and design: L Saba, SS Sanagala, SK Gupta, VK Koppula, JS Suri; (II) Administrative support: SK Gupta, AM Johri, N Sharma, JS Suri; (III) Provision of study materials or patients: JS Suri; (IV) Collection and assembly of data: A Nicolaides; (V) Data analysis and interpretation: AM Johri, NM Khanna, S Mavrogeni, JR Laird, G Pareek, M Miner, PP Sfikakis, A Protogerou, DP Misra, V Agarwal, AM Sharma, V Viswanatha, VS Rathore, M Turk, R Kolluri, K Viskovic, E Cuadrado-Godia, GD Kitas, A Nicolaides, JS Suri; (VI) Manuscript writing: All authors; (VII) Final approval of manuscript: All authors.

Correspondence to: Dr. Jasjit S. Suri, PhD, MBA, FIEEE, FAIMBE, FAIUM, FAPVS. Fellow, American Institute of Medical and Biological Engineering, Fellow, American Institute of Ultrasound in Medicine, Fellow, Asia Pacific Vascular Society, Stroke Monitoring and Diagnosis Division, AtheroPoint ${ }^{\mathrm{TM}}$, Roseville, Roseville, CA 95661, USA. Email: jasjit.suri@atheropoint.com.

\begin{abstract}
Cardiovascular disease (CVD) is one of the leading causes of morbidity and mortality in the United States of America and globally. Carotid arterial plaque, a cause and also a marker of such CVD, can be detected by various non-invasive imaging modalities such as magnetic resonance imaging (MRI), computer tomography (CT), and ultrasound (US). Characterization and classification of carotid plaque-type in these imaging modalities, especially into symptomatic and asymptomatic plaque, helps in the planning of carotid
\end{abstract}

^ ORCID: Skandha S. Sanagala, 0000-0003-3535-5613; Suneet K. Gupta, 0000-0002-0757-1290; Vijaya K. Koppula, 0000-0002-47089538; Amer M. Johri, 0000-0001-7044-8212; Narendra N. Khanna, 0000-0002-6935-0039; Sophie Mavrogeni, 0000-0003-1089-7766; John R. Laird, 0000-0003-2095-2191; Gyan Pareek, 0000-0002-2750-0967; Martin Miner, 0000-0001-6813-9242; Petros P. Sfikakis, 0000-0001-5484-2930; Athanasios Protogerou, 0000-0002-3825-532X; Durga P. Misra, 0000-0002-5035-7396; Vikas Agarwal, 0000-00024508-1233; Aditya M. Sharma, 0000-0003-1756-2504; Vijay Viswanathan, 0000-0001-9116-3937; Vijay S. Rathore, 0000-0003-4315-5072; Monika Turk, 0000-0002-3326-2766; Raghu Kolluri, 0000-0002-4374-7513; Klaudija Viskovic, 0000-0002-5927-3201; Elisa CuadradoGodia, 0000-0002-9147-875X; George D. Kitas, 0000-0002-0828-6176; Neeraj Sharma, 0000-0001-6610-7888; Andrew Nicolaides, 00000003-3912-7394; Jasjit S. Suri, 0000-0001-6499-396X 
endarterectomy or stenting. It can be challenging to characterize plaque components due to (I) partial volume effect in magnetic resonance imaging (MRI) or (II) varying Hausdorff values in plaque regions in CT, and (III) attenuation of echoes reflected by the plaque during US causing acoustic shadowing. Artificial intelligence (AI) methods have become an indispensable part of healthcare and their applications to the non-invasive imaging technologies such as MRI, CT, and the US. In this narrative review, three main types of AI models (machine learning, deep learning, and transfer learning) are analyzed when applied to MRI, CT, and the US. A link between carotid plaque characteristics and the risk of coronary artery disease is presented. With regard to characterization, we review tools and techniques that use AI models to distinguish carotid plaque types based on signal processing and feature strengths. We conclude that AI-based solutions offer an accurate and robust path for tissue characterization and classification for carotid artery plaque imaging in all three imaging modalities. Due to cost, user-friendliness, and clinical effectiveness, AI in the US has dominated the most.

Keywords: Stroke; cardiovascular disease (CVD); carotid imaging; magnetic resonance imaging (MRI); computer tomography (CT); ultrasound (US); artificial intelligence; risk stratification

Submitted Nov 26, 2020. Accepted for publication Feb 25, 2021.

doi: $10.21037 /$ atm-20-7676

View this article at: http://dx.doi.org/10.21037/atm-20-7676

\section{Introduction}

Cardiovascular disease (CVD) is the leading cause of death globally, with nearly 18 million deaths annually (1). In the US alone, approximately 700,000 people die each yearthe equivalent of 1 in 4 people dying every 36 seconds $(2,3)$. CVD's leading cause is atherosclerosis, now thought to be contributed by arterial inflammation $(4,5)$. There are several risk factors for CVD and atherosclerosis, including hypertension, hyperlipidemia, diabetes, obesity, smoking, and a sedentary lifestyle $(6,7)$. The process of atherosclerosis is characterized by excessive plaque deposits in the arterial wall (8). In the case of an unstable plaque, the associated fibrous cap breaks, causing a form of thrombosis (9). The thrombosis leads to embolism, which, in turn, causes a blockage in blood flow resulting in a stroke or myocardial infarction (10).

The plaque, which forms over time, can be detected using imaging modalities like magnetic resonance (MR), computer tomography (CT), and ultrasound (US) (11). Ordinarily, it can be challenging to determine the nature of plaque. Its various components-formed at different stages during its maturation-are spatially distributed in the arterial walls. Thus, the contrast visible in standard imaging does not clearly differentiate between components $(12,13)$. This can be due to (I) partial volume effect in magnetic resonance imaging (MRI) (14) or (II) varying Hausdorff values in plaque regions with different components in CT (15), and (III) attenuation of echoes reflected by the plaque during
US causing acoustic shadowing from calcification (16). Despite these challenges, imaging modalities can still offer clues about the different components of the plaque, tapped by the software-based advanced characterization and classification techniques (17-19).

This study aims to better understand the physics of each modality and its interpretation capabilities $(20,21)$. Further, we explore how the detection algorithms detect and characterize and classify plaque tissue components, the so-called tissue characterization and classification of the carotid artery (TCCCA). This narrative review's secondary objective is to understand the clinical requirements for a reliable, accurate, fast, and user-friendly TCCCA based on Artificial Intelligence (AI).

Several attempts have been made to perform tissue characterization and classification on carotid artery plaque $(17,22)$. However, no in-depth reports have summarized advanced artificial intelligence (AI) methods for this application. TCCCA algorithms have changed over time to adapt to new technological developments $(21,23)$, such as machine learning $(24,25)$ and deep learning (26-30) in MR (31,32), CT, and US (33-40). Plaque characterization utilizing AI-based methods can assist in clinical decisions such as when to consider revascularization $(41,42)$.

\section{Search strategy}

For this review, we followed the search strategy as shown 


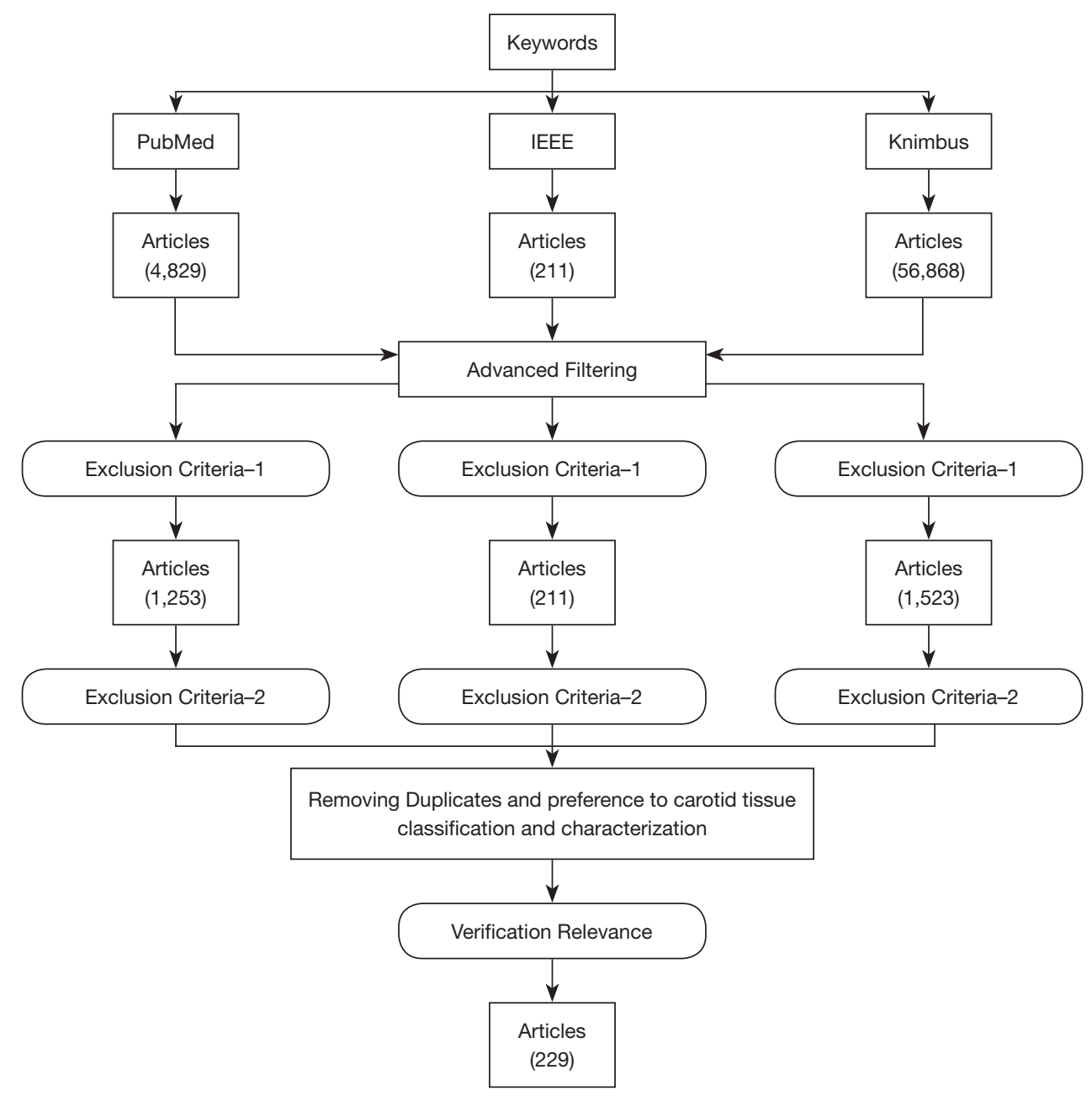

Figure 1 Search strategy showing inclusion and exclusion criteria.

in Figure 1. We used three repositories to retrieve all relevant articles related to carotid classification and characterization. Among the repositories, Knimbus is a digital library that contains well-known repositories like JSTOR, Oxford, Science Direct, Springer, Ebsco, and IEEE. We searched for "ultrasound carotid classification", "ultrasound carotid characterization", "MRI carotid classification", "MRI carotid characterization", "CT carotid classification", "CT carotid characterization", "PET carotid classification", "PET carotid characterization", "IVUS carotid classification" and "IVUS carotid characterization". After applying advanced filters such as publication year (as a range), article type, age, journal, and relevance, we found 4,829 articles in the PubMed repository, 211 in IEEE, and 56,868 in Knimbus. Using exclusion criteria-that publications must be current within ten years and specific to carotid tissue classification and characterizationand further filtering by title, abstract, and relevance, we arrived at 1,253,125, and 1,523 articles, respectively. After duplicates were removed, our final tally was 229 articles. Although we did not employ systematic review and metaanalysis (SRMA) methods, our search strategy was very similar.

\section{Search by imaging modality}

We computed the distribution of articles by modality, and Figure $2 A$ describes the articles' classification accordingly. We found that $60 \%$ dealt with US imaging techniques. US imaging is widely available, convenient, radiation-free, and low cost; thus, this technology's usage was more common and more likely to appear in the publication. Ultrasound 
A Distribution of Article by Multimodality Imaging

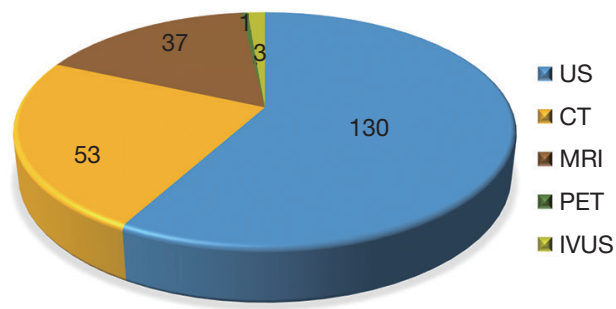

B Distribution of Article by Al Models

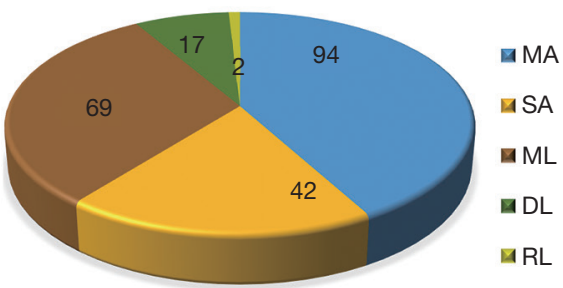

Figure 2 Classification of articles by (A) imaging modality. (B) AI techniques. MA, manual; SA, statistical analysis; ML, machine learning; DL, deep learning; RL, reinforcement learning.

imaging is the most commonly used imaging technique for applying AI methods (94.59\%), followed by CT (30\%) $(43,44)$ (yellow color) and MRI (35\%) (45) (brown color).

\section{Search by AI-models}

Figure $2 B$ shows the distribution of the AI-models applied for TCCCA. Specifically, these constituted manual analysis (MA), statistical analysis (SA), machine learning (ML), deep learning (DL), and reinforcement learning (RL). We found that $70 \%$ of the articles used MA. Machine learning (54\%) is a very common AI method in TCCCA (46). ML techniques have been used for various applications, including classifying tissue from the liver $(47,48)$, thyroids $(49)$, skin $(50)$, plaque $(19,51,52)$, and arrhythmia classification (53). ML is an older AI technique and requires manual interaction to extract features. Our search showed that the DL models had not been used often in TCCCA, as it is relatively new to medical imaging (54). Some of the key publications related to DL for medical imaging in stroke imaging are (26,55-58). RL represents $1 \%$ of all articles and one of the upcoming fields in AI-based diagnosis tools.

\section{Atherosclerosis disease and plaque formation}

Plaque development involves the engagement and transfer of smooth muscle cells (SMCs) from the media layer to the intima layer. These SMCs in the media layer move into the intima layer and form the bulk of the cellular auxiliary matrix known as the plaque creation process. These SMCs from the media layer may also enter the surface and create a layer called the fibrous cap (59), which has an elastic property that prevents it from cracking. However, the likelihood of developing a fracture also increases as this layer stiffens (60). Inflammatory cells break down SMCs to reinforce and stabilize the cap. The cap's stiffness index determines the probability of plaque breakup. Thus, determining stiffness in a stroke risk assessment is critical. This is connected to differentiating hard tissues from soft tissues $(19,25,61-63)$.

\section{Effect of plaque formation in blood vessels}

The pressure of developing plaque creates tension in the artery, which, over time, reduces the force-bearing capability of the fibrous cap. Figure 3 illustrates the plaque formation. The human carotid atherosclerotic plaque has properties such as fibrous pads, lipids, intra-plaque hemorrhage and thrombus, that shows non-linear conduct (64).

\section{Arterial stiffness}

The rupturing of this plaque then results in thrombosis or clot formation that leads to acute morbid events. Plaque formation initially begins with the deposition of small lipid and fatty materials in the intima, which can grow into complex plaque structures over time (65). The advanced plaque has a heterogeneous composition, with a lipid center, calcification, and fibrous deposits of the connective tissue (66).

\section{Cause of arterial stiffness due to atherosclerosis}

The top layer of atherosclerotic plaque, which includes SMCs, is cellular fibrosis. The elasticity of the SMCs determines the amount of force the arterial wall can withstand. The SMCs inside of the vascular walls contribute to vascular rigidity (67). During the replication process, removing the SMCs present in an artery prevents atherosclerotic plaque development. During the replication 

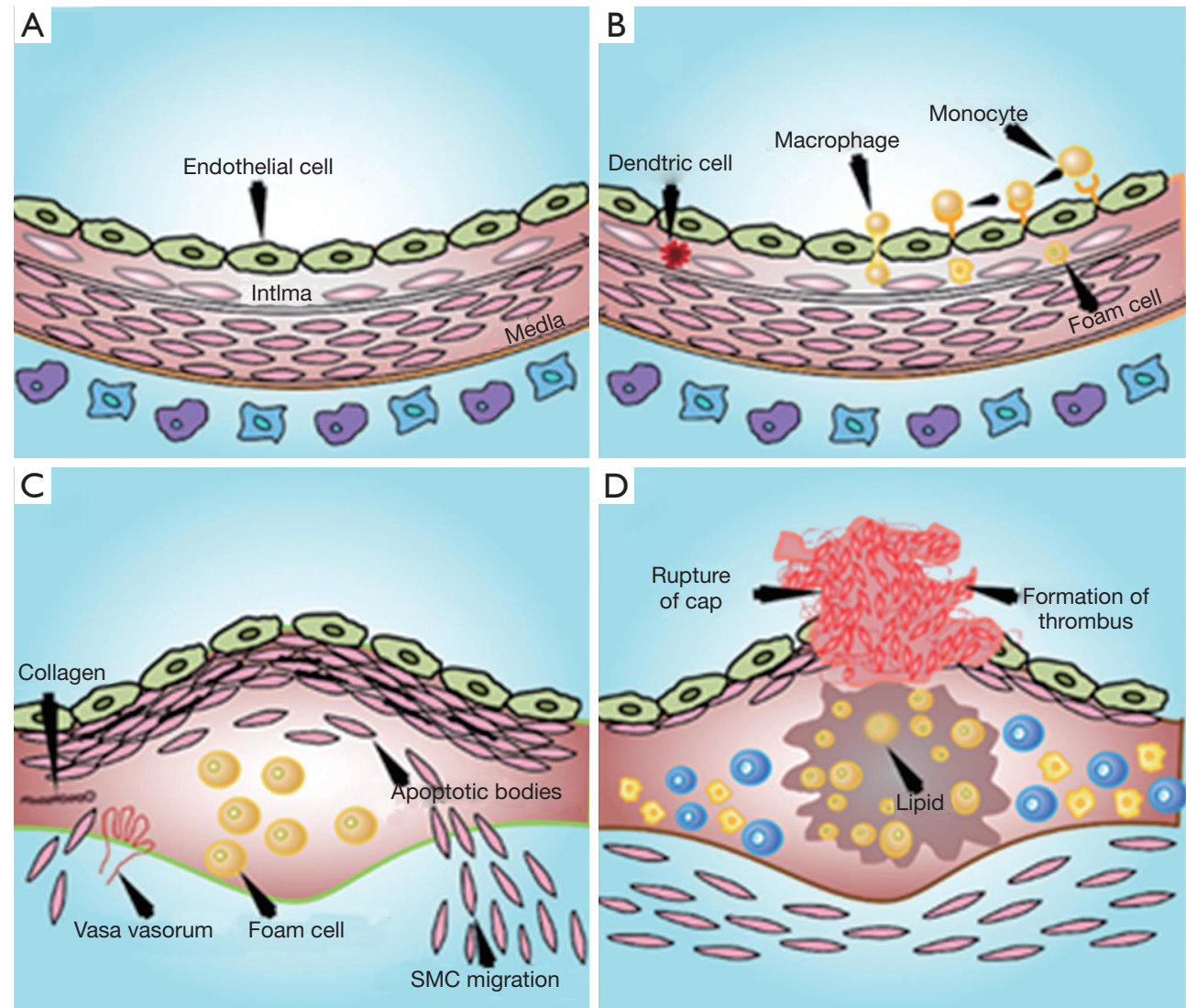

Figure 3 Stages of plaque formation and rupture. (A) Healthy artery, (B) LDL penetration via intima layer, (C) dislodging of SMC, and (D) wall rupture (courtesy of AtheroPoint, Roseville, CA, USA). SMC, smooth muscle cell.

process, the reduction or removal of the SMCs present in an artery prevents atherosclerotic plaque development. During the replication process, the small number of SMCs present in an artery prevents atherosclerotic plaque development. The small number of SMCs present in an advanced atheroma splits into deoxyribonucleic acid (DNA), which results in programmed cell death, or apoptosis. As the artery grows, inflammatory cytokines induce apoptosis, helping the SMCs to adapt. The accumulation of SMCs may be a product of cell death and replication, causing arterial stiffness (68). Previous research has suggested that increased stiffness may also be caused by changes in the collagen ratio to elastin in the arterial media's extracellular matrix (69). An example of different pathological components is shown in Figure 4.

\section{Physics of multimodality imaging for carotid plaque acquisition}

This section presents the different imaging modalities used for carotid plaque imaging (20).

\section{Magnetic resonance imaging for carotid plaque}

The MRI contains four components: (I) a magnetic coil, (II) a gradient coil, (III) a radio frequency (RF) coil, and (IV) a shim coil (70). A set of magnet coils made of superconducting metal-alloy produce powerful magnetic fields when a current passes through it. It also generates negligible heat, which is subsequently cooled by cryogenic helium. There are three concentric gradient coils located at the primary magnet coil. These gradient coils change the magnetic field's direction along the $\mathrm{x}, \mathrm{y}$, and $\mathrm{z}$-axes. $\mathrm{RF}$ coils are mounted concentric to the main magnetic field and serve as antennae to send RF energy to the tissue and receive the induced RF signals generated by the tissue (71). The MRI requires the aid of a homogenous magnetic field to localize the region of interest. Shim coils were used to homogenously adjust the magnetic 

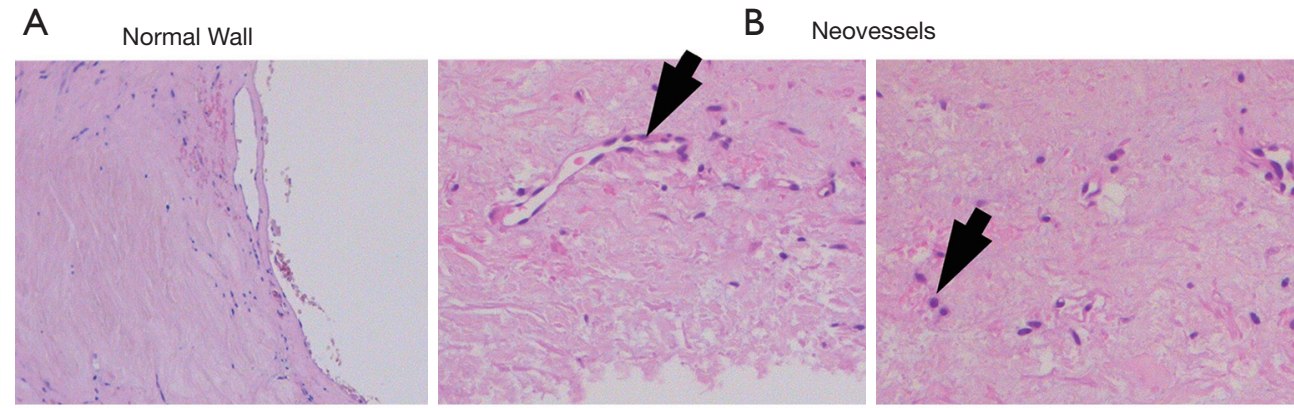

C

Calcified Plaque

D Intraplaque Hemorrhage
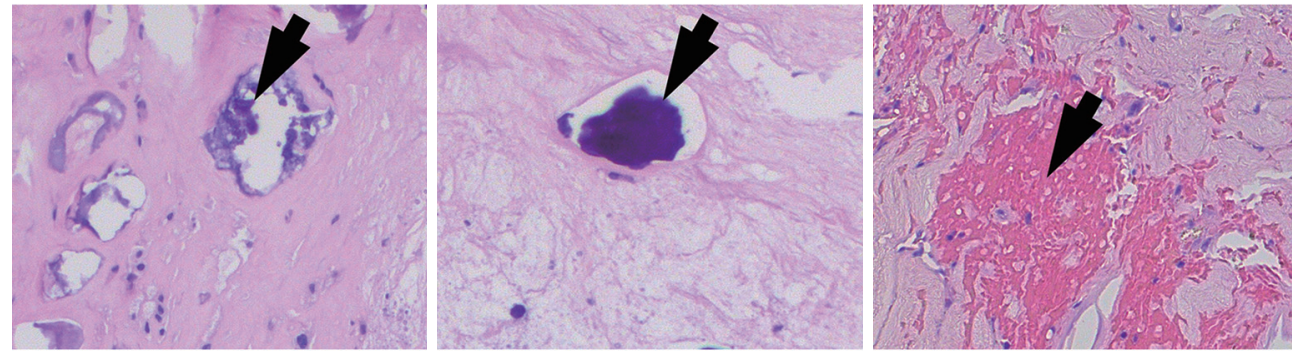

Figure 4 Pathological images were depicting different components of plaque. (A) Healthy wall, (B) neovessels, (C) calcified plaque, and (D) intraplaque hemorrhage (courtesy of Dr. Luca Saba, U of Cagliari, Italy).
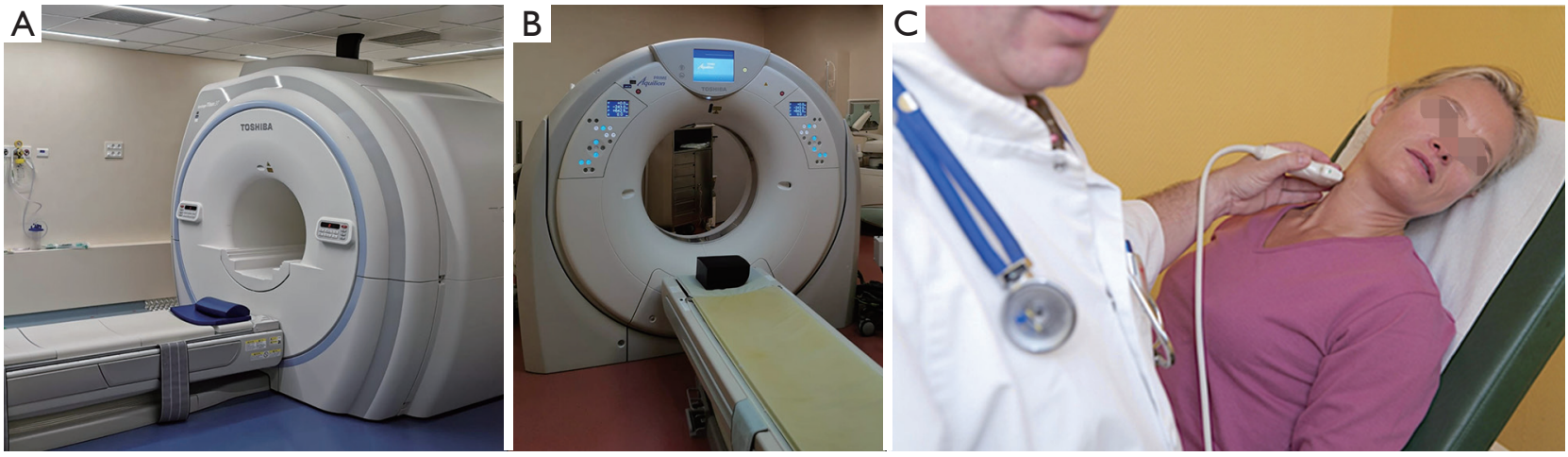

Figure 5 Imagining machines of (A) MRI, (B) CT, and (C) carotid ultrasound scanning using a linear probe (MRI/CT images-courtesy of Dr. Luca Saba, Italy, and US image, courtesy of AtheroPoint, CA, USA). MRI, magnetic resonance imaging; CT, computed tomography.

field by controlling it via the computer. The human body contains $80 \%$ water. Thus, when a patient lies in the bore of the MRI machine, the hydrogen atoms in the tissue align themselves with the magnetic field of the MRI machine. The resultant MR signals are localized and construct an image using gradient field magnets. Inhomogeneity correction in MRI for carotids can be adapted (72). Figure $5 A$ shows the MRI machine representation.

\section{Computed tomography imaging for carotid plaque}

CT imaging gives the ROI of tissue without the need to superimpose on the adjacent structure (73). It uses an $\mathrm{X}$-ray radiation attenuation coefficient (AC) when imaging organs. AC was calculated as the density of the $\mathrm{X}$-ray beam attenuated by the tissue. The CT machine mainly consists of an X-ray tube with slip-ring technology and a detector. The detector continuously rotates around the patient during the diagnosis. This technology is called Helical CT, 
which has replaced the conventional CT (step and shoot) acquisition technique. The images acquired via CT are three-dimensional and allow multiplanar reconstruction. In a CT machine, the "pitch" parameter indicates the quality of the image acquired. If the pitch is less than one, then the acquired image is considered high-quality and oversampled, but increases the patient's radiation exposure. Typically, the pitch value should be between 1 and 1.5 (74).

The thickness of the image slice depends on the X-ray collimator. The longer the collimator is exposed, the thicker the slice. However, the amount of radiation exposure increases and fails to planner reconstruction. Multiple detector array CT scanners overcome this problem. These scanners contain multiple detector arrays, and the collimator spacing is quite wide. Thus, it improves the imaging quality and slice thickness. Dual-source CT scanners have recently been developed, containing dual X-ray sources and detectors; these detectors are perpendicular to each other and rotate around the patient body. This setup cuts the acquisition time in half while still producing high-quality images.

A study was conducted recently that compares the image quality and radiation dose delivered to patients during computed tomography (CT) angiography (CTA) of the supra-aortic arteries using two single-source (SS) and two dual-energy (DE) CT scanners (75). Figure 5B illustrates a typical CT machine. Several types of energy levels are sometimes considered for the optimization of carotid plaque characterization (76).

Several applications for carotid plaque characterization and microbleeds were shown in CT $(77,78)$. Semi-automated wall measurements were computed in CT. Baradaran et al. (79) recently showed the calcium volume measurement using 74 CT. Saba et al. (80) showed that the color scale instead of the conventional grayscale improves the diagnostic confidence, accuracy, and inter-observer agreement of the readers, particularly junior ones, in the diagnosis of internal carotid artery dissection on non-contrast CT.

\section{Ultrasound imaging for carotid plaque}

Ultrasound machines have a duplex scanner and a transducer with a linear broadband width of 4-7 MHz (multifrequency) and a resolution of 20 pixels $/ \mathrm{mm}$. Ultrasounds operate on the principle of the piezoelectric effect (81). The machine contains a transducer, a CPU, and a monitor. The transducer contains piezoelectric crystals, which act as the senders and receivers of ultrasound signals.
The piezoelectric crystals generate sound waves when a current flows through them, and they generate a voltage when they receive a vibration (ultrasonic wave) (5). When the ultrasound waves fall on the ROI tissue, the tissue reflects the ultrasonic waves (echo), the transducer catches the reflected signals. It then constructs and displays an image in the monitor based on these signals.

Some prerequisites are necessary for successful image normalization. The following prerequisites were carried out in this study: (I) dynamic range was adopted. (II) Frames were averaged. (III) The time gain compensation curve that was sloping through the tissues was positioned vertically through the vessel's lumen because the ultrasound beam was not attenuated when it passed through the blood. This was done to ensure that the adventitia of the anterior and posterior walls had similar brightness. (IV) The gain was adjusted. (V) Post-processing was done using a linear transfer curve. (VI) The ultrasound beam was maintained at an angle of 90 degrees to the arterial wall. (VII) The minimum depth was used to ensure that the plaque occupied a large part of the image. (VIII) The probe was adjusted so that the adventitia adjacent to the plaque was visible as a hyperechoic band used for normalization. The ultrasound machine at Atheropoint ${ }^{\mathrm{TM}}$ is shown in Figure 5 C.

The MRI cross-sectional scans of the carotid plaque are shown in Figure $6 A$ (82). The CT cross-sectional scans of the carotid plaque are shown in Figure $6 B(83)$. Figure $6 C$ presents the plaque acquisition demonstrate in the US (40). Figure 7 provides a comparison of intraplaque hemorrhage (IPH) in a male patient's CTA and MRI and histopathology (74).

Other modalities, like molecular imaging positron emission tomography (PET), can also study plaque biology (85) and find the sequential stages of inflammation in atherosclerosis. Intra-vascular ultrasounds yield promising results for understanding vulnerable carotid features. Also, IVUS was perceived as one of the best imaging technique for studying carotid (86).

\section{Challenges in plaque classification/characterization and making a case for $A I$}

The biggest challenge in plaque characterization is the fuzziness in the walls' intimal-media region, immaterial of the imaging modality. This causes validations in contrast; for example, in ultrasound, symptomatic plaques are hyperechoic (bright), having a high-lipid core, low calcium, and are vulnerable, unlike asymptomatic plaque, which is hypo- 

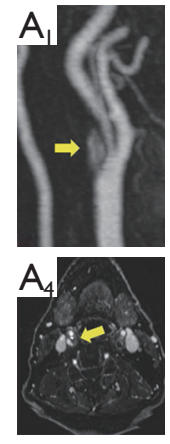
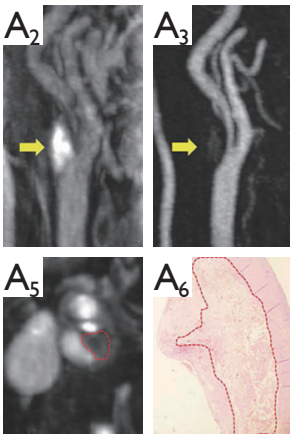
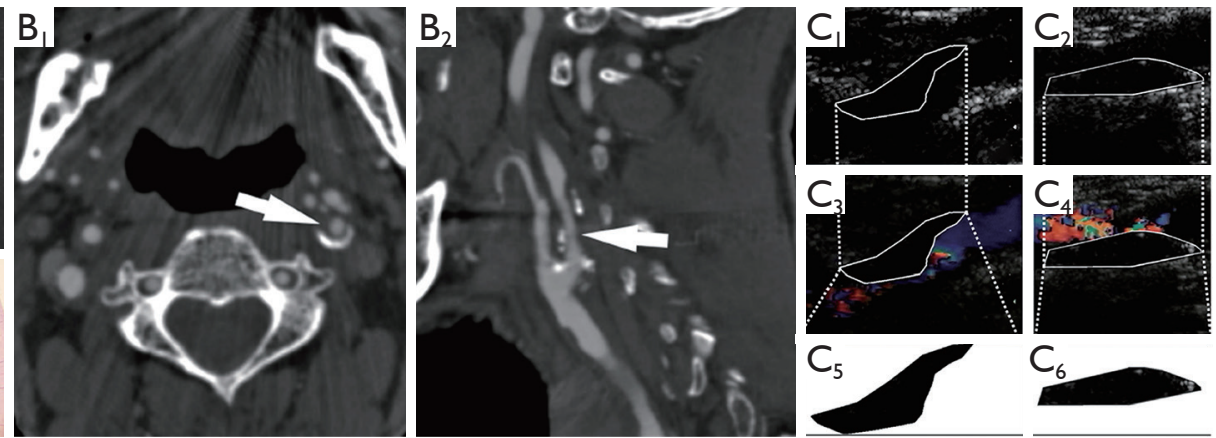

Figure 6 Carotid plaque scans from (A) MRI scans of ICAP; the yellow arrow represents intraplaque hemorrhage (A1) hyperintense on T1-wt MRI, (A2) hypointense on TOF, (A3) hyperintense on T1-wt MRI after contrast medium injection in sagittal, and (A4) axial view, (A5, A6); the red area represents the intraplaque hemorrhage in the axial section of T1-wt MRI. (B) [courtesy of (82)], (B) CT scans of the ICAP; the white arrow represents the plaque developed (B1) axial, and (B2) sagittal scan [courtesy of (83)]. (B) US scans of ICAP, (C1, C2) represent the symptomatic plaque images, (C3, C4) corresponding color Doppler images, and (C5, C6) delineated plaques (40) (courtesy of AtheroPoint, Roseville, CA, USA) [Permitted to reproduce Figure (A) and (B)]. MRI, magnetic resonance imaging; ICAP, internal carotid artery plaque.
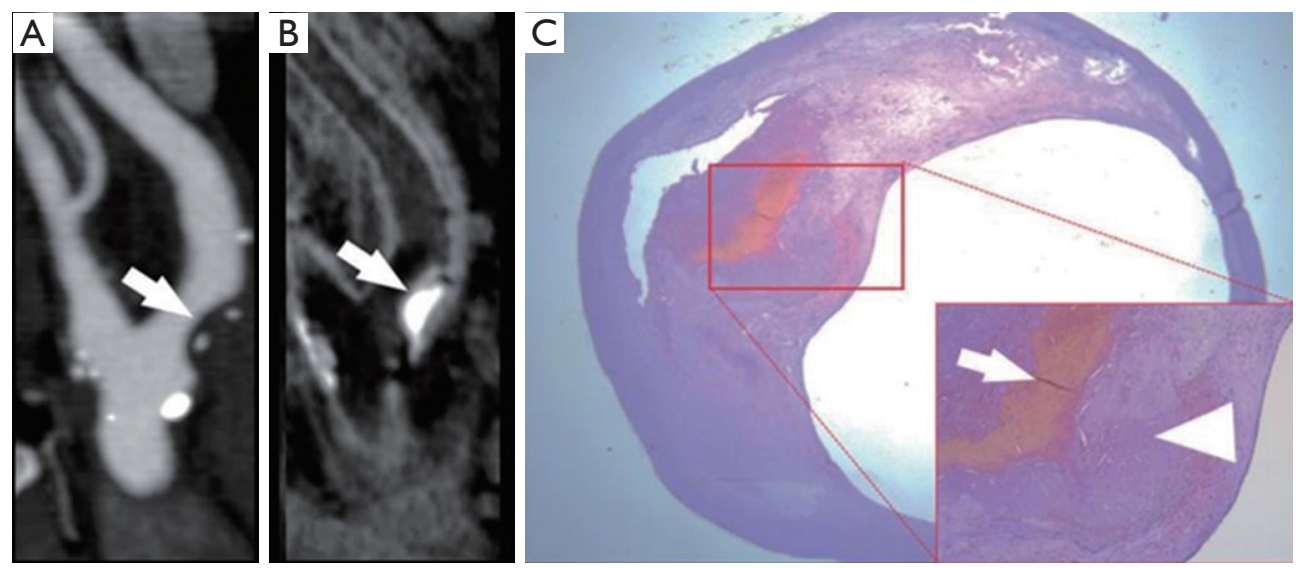

Figure 7 Comparison of the IPH visualization in (A) CTA, (B) MRA, and (C) the histopathology section; the white arrow represents IPH [source (84), Permitted to reproduce]. IPH, intraplaque hemorrhage; CTA, computed tomography angiography; MRA, magnetic resonance angiography.

echoic, having poor lipid core, stable, and appears dark in ultrasound scans. Thus, characterizing this plaque with the naked eye is unreliable and subject to error and has interobserver variability $(87,88)$.

With the help of computer vision techniques and artificial intelligence, however, we can identify the plaque $(89,90)$, in spite of variations and fuzziness in pixel distribution to be non-linear (91). Such images require machines with greater learning capabilities. Recent advancements in machine learning techniques offer precisely this; now, plaques can be identified, classified, and characterized using the "handcrafted" feature (19,38,40,52,61,62,92-99). These methods achieve the objective but lack reliability and stability due to variation in the features and performance of different AI models. Deep learning technology, meanwhile, can fill in the gaps using automatic feature extraction $(55,100)$ through a convolutional neural network $(\mathrm{CNN})$ that allows us to characterize plaques (55).

\section{Artificial intelligence architectures}

The nature of AI architecture design has gradually evolved 
over time, starting from manual methods and signal processing methods and eventually leading to learning methods (so-called AI models). Thus, we have divided this section into AI architectures by modality for TCCCA.

\section{Manual, semi-automated, and statistical models using $M R, C T$, and the US}

Several authors have used manual and statistical methods for TCCCA (101-108). In van Ingersleben et al. (109), the radiologist characterized the carotid plaque at the bifurcation by identifying calcium, lipid deposits, and fibrous plaques, based on ex-vivo imaging. Using MRI, Yuan et al. (32) showed the importance of carotid MR imaging and evaluated the plaque morphology and its composition rather than lumen stenosis. Chu et al. (110) showed that carotid lesion characterization requires the quantitative measurement of the carotid artery wall thickness. The authors followed the AHA guideline for characterization of the carotid lesions, namely type I-II, considered as near-normal wall thickness without calcification; type III, considered as diffuse wall thickening or small eccentric plaque without calcification; type IV-V, considered as a plaque with a lipid-rich necrotic core surrounded by fibrous tissue with possible calcification; type VI, considered as a complex plaque with a possible surface defect, hemorrhage, or thrombus; type VII, considered as calcified plaque; and type VIII, fibrotic plaque without a lipid core and with possible small calcifications. In MRI, Zhao et al. (111) showed the role of high-risk plaque (HRP), defined as plaques having luminal surface disruption or a lipid-rich necrotic core occupying $>40 \%$ of the wall, or intraplaque hemorrhage compared to stenosis in 1,047 recruited subjects. The authors demonstrated that HRP using carotid MRI was $1.5 x$ greater than stenosis of $\geq 50 \%$. These surface characteristics that originated from the composition had a greater stroke risk, unlike the lumen constriction.

In CT, Wintermark et al. (112) showed a CT-based degree of stenosis (DoS) by comparing a semi-automated method against a neuroradiologist's visual assessment using Kappa statistics (so-called gold standard), yielding an agreement of $0.918(\mathrm{P}<0.001)$. Further, Chien et al. $(113)$ conducted a cross-sectional study in which the authors characterized the CTA plaque by correlating the symptoms (or risk factors). These symptoms included hypertension, age, and carotid bruit. Univariate and multivariate analyses were performed, demonstrating the $\mathrm{P}$ value close to 0.0 . Wintermark et al. (114) conducted a cross-sectional study to explore the
CT-based features of carotid plaque (namely lumen area, wall volume, number of calcium clusters, fibrous cap thickness, number of lipid clusters, and the location of the largest lipid clusters) against the two types of strokes (acute carotid stroke patients and non-acute carotid stroke patients). The Causative Classification System identified the gold standard for Ischemic Stroke with the help of a neuroradiologist. After undergoing TCCCA of the CT carotid wall, acute carotid stroke patients demonstrated a significantly greater difference between the CA wall ipsilateral's appearance to the side of their infarct $(\mathrm{OR}>1.0$ with $\mathrm{P}=0.0)$ when compared against the non-acute carotid stroke patients. Li et al. (115) characterized several CT carotid wall-based features, namely, (I) maximum plaque height (MPH), (II) soft plaque, and (III) carotid arterial stenosis (DoS) and associated with 10year atherosclerotic cardiovascular disease (ASCVD) (into two groups having a cut-off of $7.5 \%$ ), thereby showing concordance results but not perfect overlap.

In the US, Griffin et al. (116) identified high-risk patients by characterizing the carotid plaque in the US using the juxtraluminal black area (JBA) as a risk predictor in the univariate logistic regression paradigm. The ground truth had three classes based on mild, moderate, and severe carotid stenosis. SPSS 10.0 was used to compute the regression coefficient, followed by the odds ratio (OR). The authors demonstrated by changing the cut of JBA $>8 \mathrm{~mm}^{2}$ and GSM $<15$, they were able to detect identify a highrisk group of 188 plaques that contained $142(77 \%)$ of the 185 symptomatic plaques [odds ratio (OR), 6.7; 95\% confidence interval (CI), 4.08-10.91, $\mathrm{P}<0.001]$, (sensitivity: $77 \%$; specificity $66 \%$; positive predictive value $75 \%$; negative predictive value $68 \%$ ). In another work, Nicolaides et al. (17) demonstrated that a number of baseline clinical characteristics and ultrasonic plaque features are independent predictors of subsequent ipsilateral cerebral or retinal ischemic (CORI) events. The author uses "Plaque Texture Analysis Software v3.4" to calculate the risk of the individual patient based on the Cox model. In (117), the same authors discussed the image acquisition technique for 3-D ultrasound image reconstruction from a series of 2-D ultrasound scans using "Philip QLAB and Plaque Texture Analysis Software v3.4". The authors extracted the GSM, JBA, plaque area, and SGLDM-difference entropy (SGLDM-DE) from the 2-D and 3-D images. The authors found no linear relationship between 2-D GSM and its corresponding 3-D GSM $(\mathrm{P}=0.24)$, and there is a moderate relation between 2-D JBA and its corresponding juxtraluminal black volume (JBV). The authors found that 


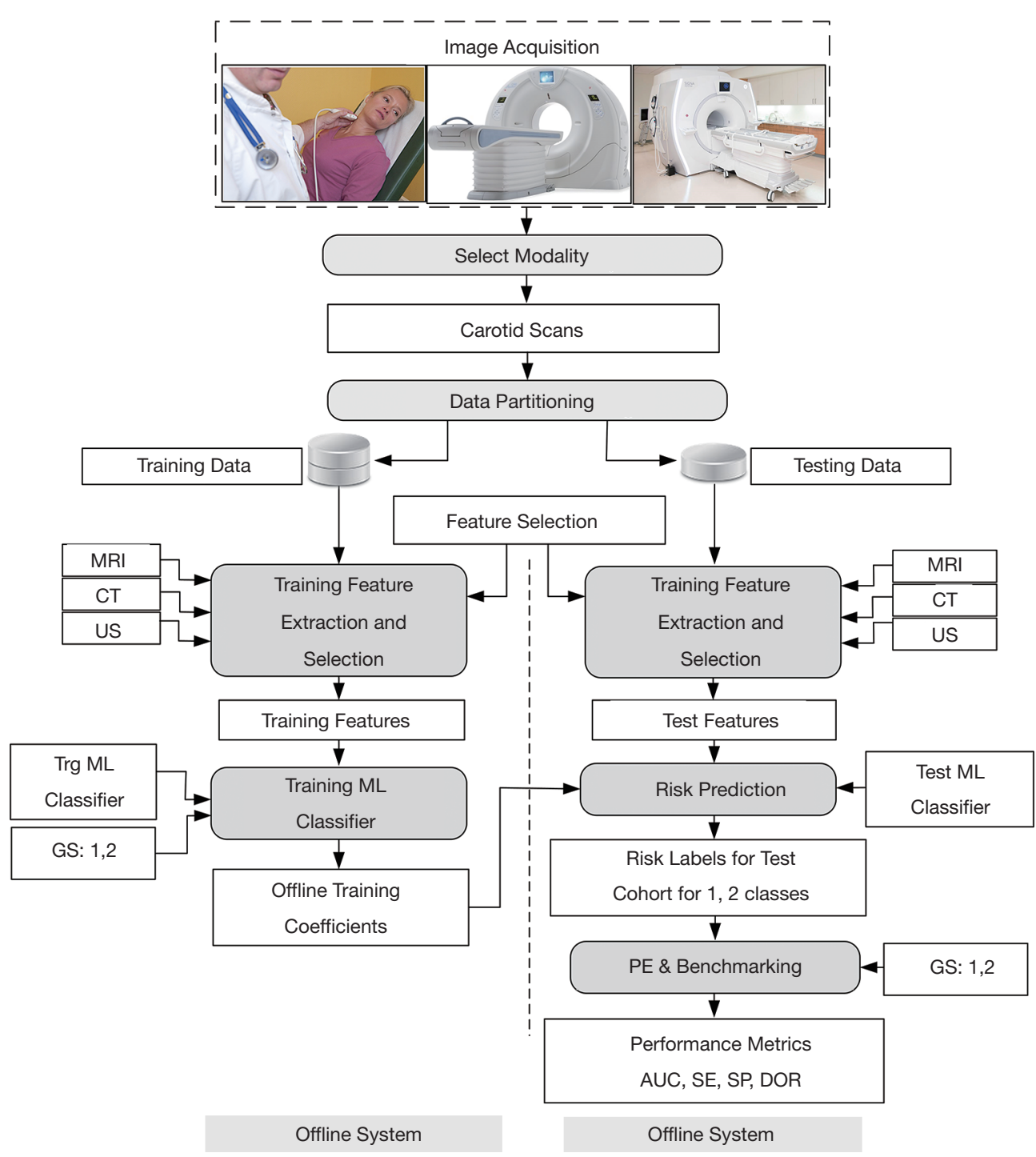

Figure 8 Generalized global ML architecture for tissue classification using MRI, CT, and US (courtesy of AtheroPoint, Roseville, CA, USA). GS, gold standard; PE, performance evaluation; AUC, area under the curve; SE, sensitivity; SP, specificity; DOR, diagnostic odds ratio; MRI, magnetic resonance imaging; CT, computed tomography.

JBA and JBV are higher in symptomatic than asymptomatic $(\mathrm{P}=0.004$ and $\mathrm{P}=0.002)$. The authors observed a good linear relation between 2-D plaque area and 3-D plaque volume. It is observed that plaque area is higher in symptomatic plaque $(\mathrm{P}=0.18)$, but in the plaque, the volume is not showing that characteristic $(\mathrm{P}=1.0)$. SGLDM-DE is showing a poor relationship between 2-D and 3-D. Loizou et al. (118) extracted the same texture features from plaque components and identified the stroke risk by measuring the statistical difference of the symptomatic and asymptomatic cases. The authors showed 71 texture features to be different between symptomatic and asymptomatic. Jamthikar et al. (119) from the AtheroPoint team studied a narrative review on artificial intelligence techniques in ultrasound imaging modality for tissue classification, characterization, and CVD risk assessment.

ML has started to become more prevalent in the field of AI, as it provides a way to extract knowledge from different covariates of a cohort. This knowledge can be utilized to assess the non-linearity between predictors and distinguish cardiovascular predictors from cerebrovascular predictors. Figure 8 depicts the general architecture of 
the ML system that consists of offline and online systems. The risk stratification was predicted online, as shown in Figure 8 .

\section{Machine learning for plaque characterization in MRI}

Winn et al. (31) characterized 20 carotid fibrous caps in T2weighted MRI using four observers (radiologists). The ROI was generated by comparing T2-weighted MRI against histological images taken from carotid endarterectomy. The AUC came out to be 0.75 . The authors further characterized fibrous cap or rupture, having detection and characterization evaluated as $90 \%$ and $98 \%$, respectively. Validation of 3-D ultrasound imaging for plaque vulnerability is crucial for clinical viability. Chiu et al. (120) did exactly that by registering 3-D segmented carotid ultrasound against 3-D MRI using a surface-based image registration algorithm, as shown in previously developed methods $(121,122)$. The authors demonstrated an average error rate of less than $1 \mathrm{~mm}$ in the in-vivo study and $0.3 \mathrm{~mm}$ in the phantom study. The carotid artery geometry requires imaging more than $3 \mathrm{~cm}$ in length from the collar bone to the jaw. Not always, the plaque can be captured in the wall of these arteries. This is because CCA bifurcates into ICA and ECA. As a result, some plaque is identified, some partially identified, and some are entirely missed. Murata et al. (123) designed a motion-sensitized driven equilibrium prepared rapid gradient echo (3-D MERGE) acquisition protocol to characterize carotid plaque using black blood MRI. The authors analyzed 97 subjects consisting of 194 carotid arteries (70 men and 27 women, mean age 60 years). The authors identified 136 plaques; 68 (50\%) were within, $46(33.8 \%)$ were partially outside, and 22 (16.2\%) were entirely outside of 2-D MRI coverage. Guan et al. (124) developed two kinds of segmentation algorithms, namely structural SVM (SSVM) and Bayes segmentation, to detect the internal wall. The authors compared the two segmentation algorithms' results and demonstrated SSVM to be more robust than Bayes. The authors achieved a misclassification rate of $16.9 \%$ in Bayes and 9.6\% in SSVM.

Zhang et al. (125) assessed the importance of using simultaneous non-contrast angiography and intraplaque hemorrhage (SNAP) to detect the lipid-rich/necrotic core (LRNC) from among 1,436 scans obtained by 3T MRI and 3-D SNAP sequence. The authors compared the classification accuracies of manually segmented plaques with plaques segmented by SNAP. The accuracies of NB, SVM, RF, gradient boosting decision tree (GBDT), and ANN were higher than $88 \%$ when the manually segmented plaque was used. However, when SNAP was used, the same classifiers achieved higher than $78 \%$ accuracy $(\mathrm{P}<0.0001)$. Studies (126) have been conducted for the registration of MRI volumes using a different protocol for plaque characterization.

\section{Macbine learning for plaque characterization in CT}

Zhu et al. (127) investigated CT Angiogram (CTA) for quantitative features to predict 10 years' worth of atherosclerotic cardiovascular disease (ASCVD) risk using 117 CTA scans. The authors built two semi-automated linear regression models with continuous and dichotomous features. The models yielded ASCVD risk scores of $18.87 \% \pm 13.26 \%$ and $18.39 \% \pm 11.6 \%(\mathrm{P}<0.0001)$, and the mean biases between observed ASCVD and predicted ASCVDs were $-1.954 \% \pm 10.88 \%$ and $-1.466 \% \pm 12.04 \%$, respectively. The most accurate prediction for the ischemic stroke depends on the optimal spatial coverage of the carotid plaque. Arora et al. (128) studied the optimal coverage area required to distinguish between stroke and non-stroke patients. The authors found that $20 \mathrm{~mm}$ coverage on each side of the carotid bifurcation offers optimal results. Further, the authors used a CT-automated classification algorithm, multivariate, and univariate analysis on different components of plaque obtained from 136 patients.

He et al. (129) used optical coherence tomography (OCT) to quantitatively assess the plaque vulnerability of 31 patients based on ex-vivo carotid plaques. The authors accurately classified the fibrous, calcified, and lipid core components of plaque by extracting texture features and pixel-wise features using an RF classifier. Accuracies of $80 \%, 62 \%$, and $83.1 \%$ were achieved with sensitivity and specificity (\%) combinations of $(80.5,91.2),(64.7,90.7)$, and (87.5, 80.2). Acharya et al. (92) classified CT carotid artery images from 20 patients as symptomatic and asymptomatic by extracting LBP features and wavelet features with SVM. Their classifications were $88 \%$ accurate. Further, they characterized the plaque using the Atheromatic index (AtheroPoint, CA, USA), as shown in Figure $9 A$.

\section{Machine learning for plaque characterization in the US}

Most studies carried out within the ML framework have used ultrasounds for TCCCA. Such studies are typically divided into two parts: one based on grayscale information and another based on non-grayscale information (so-called point-based 

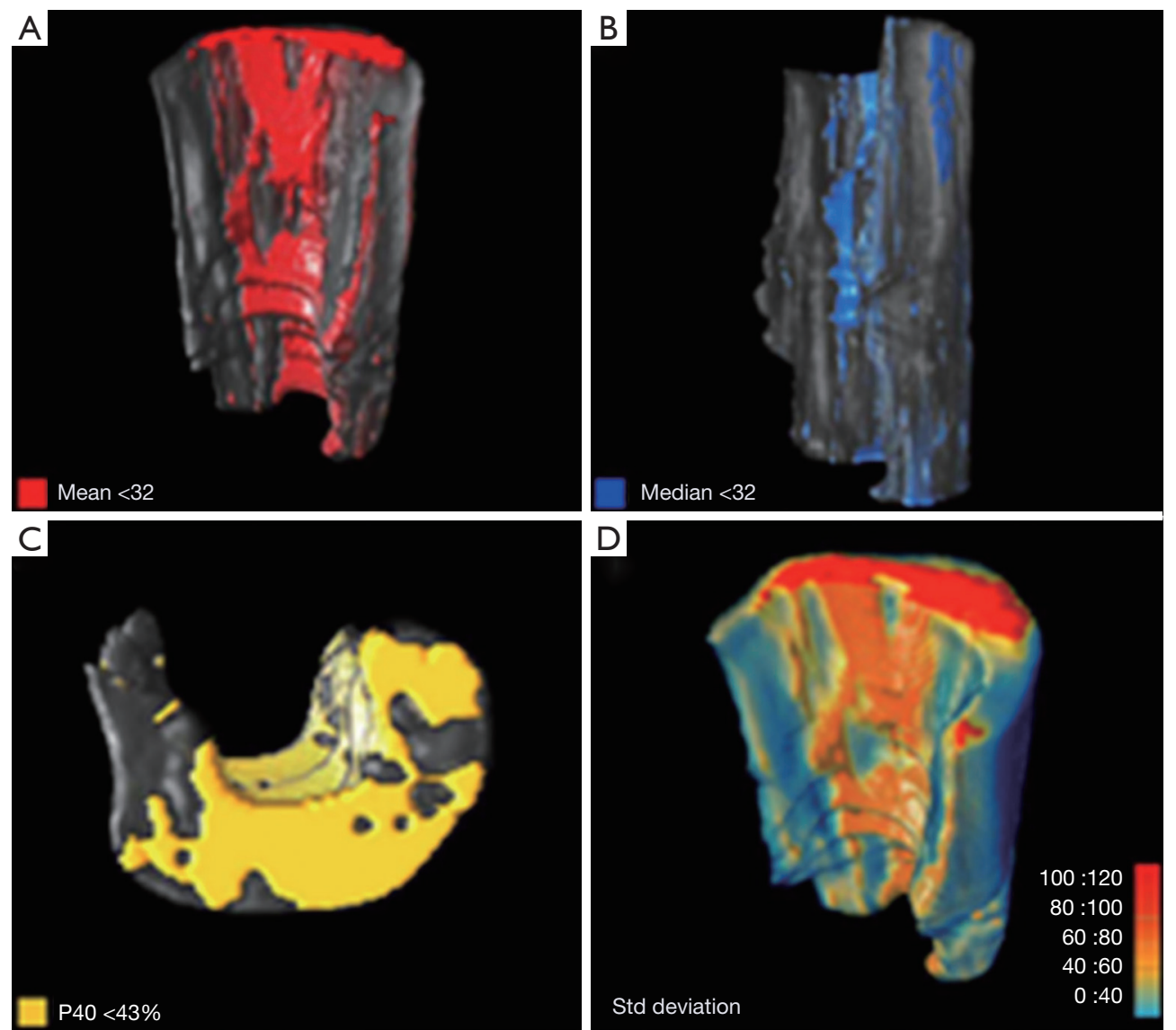

Figure 9 Local assessment of plaque echo-morphology, in terms of (A,B,C) hypoechogenicity and (D) heterogeneity [Source (33), Permitted for reproduction].

information). Several ML methods have been tried on various organs such as thyroid gland (130-132), liver $(47,48,133)$, ovarian (134-137), carotid plaque characterization $(98,138)$, coronary $(139,140)$, stroke risk $(19,52,141)$, lung tissue characterization (142).

3-D carotid plaque characterization was tried by Seabra et al. (143) mainly to overcome the weakness due to subjectivity with data acquisition and operator-dependent selection. In their characterization model, the authors applied a labeling procedure using graph cuts, which allow them to identify, locate, and quantify vulnerable plaque. The authors tested their method on five patients who had undergone 2-D ultrasound scans and validated their synthetic data method. The same authors in (33) proposed a Bayesian technique for estimating the volume inside plaque by removing speckle noise in the ROI. The authors compared the proposed method with a gold standard, and the quantified volumes P40 (40\% hypoechogenic voxels) were 56.76 and $50.62 \mathrm{~mm}^{3}$, respectively, while GSM $<32$ were 49.31 and $44.29 \mathrm{~mm}^{3}$, respectively. The author's assessment of ecomorphology is shown in Figure 9.

Seabra et al. (144) proposed a classification model using an ML classification technique (Adaboost) and tested it on 146 US scans using leave one patient out crossvalidation protocol (LOPO CV). The authors extracted the texture features fused with clinical information, achieving an accuracy rate of $99.2 \%$ and $100 \%$ sensitivity. Afonso et al. (145) developed a computer-aided diagnosis tool for measuring plaque rupture risk using an activity index and an enhanced activity index (EAI). The authors were able to characterize the plaque echogenicity using the EAI.

Christodoulou et al. (97) classified 330 carotid ultrasound scans (CUS) as symptomatic and asymptomatic using a self-organizing map (SOM) and k-nearest neighbor through which they extracted texture features spatial gray level dependence matrix (SGLDM), gray level difference 
Table 1 The literature on TCCCA using ML techniques

\begin{tabular}{lccc}
\hline SN\# & ML model & Modality & References \\
\hline 1 & SVM & US & $(19,34-40,61,94-96,146,149-151)$ \\
2 & KNN & US & $(13,40,95,97,98,152)$ \\
3 & Adaboost & US & $(61)$ \\
4 & UAI & US & $(145,153)$ \\
5 & PNN & US & $(34,37,40,95,149)$ \\
6 & Adaboost & US & $(144,154)$ \\
7 & SOM & US & $(97,152,155)$ \\
8 & ANN & US & $(35,156-158)$ \\
9 & NB & US & $(35)$ \\
10 & LR & US & $(36)$ \\
11 & LDA & US & $(95)$ \\
12 & QDA & US & $(95)$ \\
13 & DT & US & $(95)$ \\
14 & RF & MRI & $(159)$ \\
15 & & CT & $(129)$ \\
16 & DT, NB, RF, & CT & $(160)$ \\
17 & LR, NN & & CT \\
\hline
\end{tabular}

TCCCA, tissue characterization and classification of the carotid artery; ML, machine learning; US, ultrasound; MRI, magnetic resonance imaging; $\mathrm{CT}$, computed tomography.

statistics (GLDS). With the help of first-order statistics, the researchers achieved a 70\% diagnostic yield (DY). The author (25) extracted ten different Law texture energy features and fractal dimension features from CUS. The author then fed these features into SOM and $k-\mathrm{NN}$ for classification, achieving $73.1 \%$ and $68.8 \%$ accuracy rates, respectively. Loizou et al. (146) proposed a classification model to investigate the texture feature variability obtained from 120 longitudinal ultrasound videos in M-mode during the cardiac cycle. The authors automatically segmented the plaque using a snake algorithm. Further, the authors classified it as either systolic or diastolic using SVM with texture features, achieving $100 \%$ accuracy and a ROC of 1.0. Doonan et al. (147) studied the correlation between the texture and echo density features of plaque based on 104 bilateral US scans. The authors extracted the features using commercial "plaque texture analysis software" and PCA. The correlation between these two types of features was between 0.211 and $0.641(\mathrm{P}<0.0001)$.

Our group had implemented several carotid characterization techniques in the machine learning framework. Acharya et al. (40) proposed a classification technique based on 346 US scans in which texture, LBP, and Law's Texture Energy (LTE) features were extracted using SVM. The authors achieved an accuracy of $83 \%$, a sensitivity of $84.4 \%$, and a specificity of $79.7 \%$. In this study (93), the authors extracted texture-based features from 99 US scans and fed them into SVM using RBF kernel. This method achieved an accuracy of $91.7 \%$, a $97 \%$ sensitivity, and a specificity of $80 \%$. The authors then characterized the plaque using an asymptomatic carotid index. In this study (94), the authors extracted discrete wavelet transform features and texture features. They then fed the features into SVM with a polynomial kernel of order 2, achieving $83.7 \%$ accuracy, a sensitivity of $80 \%$, and a specificity of $86.4 \%$. Molinari et al. (95) proposed a data mining framework for classifying symptomatic and asymptomatic plaque using bi-dimensional empirical mode decomposition and entropy features. They achieved an accuracy of $91.43 \%$. The researchers also studied the relationship between coronary plaque and carotid IMT $(139,148)$. Table 1 lists the reviewed literature regarding ML techniques for TCCCA.

\section{Deep learning strategies using MRI, CT, and the US}

Deep learning (DL) methods have changed the paradigm of AI. They have accelerated the application of AI techniques in medical imaging owing to the flexible and reliable strategies associated with them. A significant drawback of the ML model was solved by using automatic feature extraction via convolution neural networks $(\mathrm{CNN})$. In the DL model, all acquired grayscale images are fed into the convolution layers. This process is followed by maxpooling/average pooling layers for automatic feature extraction. These extracted features are subsequently fed into a fully connected network for classification and risk assessment. The global architecture of a DL system is shown in Figure 10A. DL models learn complex patterns from input training images and then use these patterns to predict target labels. There are several models besides $\mathrm{CNN}$ that are popular in medical imaging. Some of these are the U-Net, Autoencoders, recurrent neural networks (RNNs), long short-term memory (LSTM), Regionbased Convolutional Neural Networks (R-CNN), and Mask R-CNN. U-Net (a combination of encoders and decoders) plays an important role in the segmentation and classification processes $(55,161-163)$. Figure $10 B$ shows the 
A

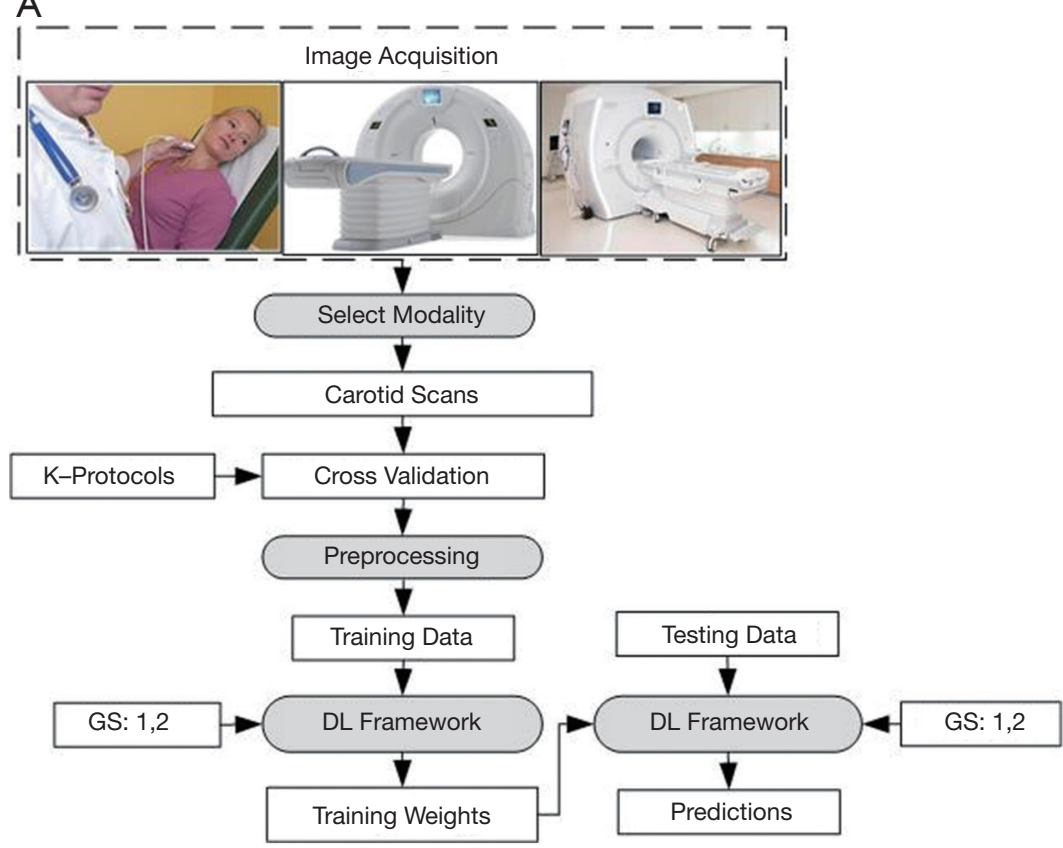

B

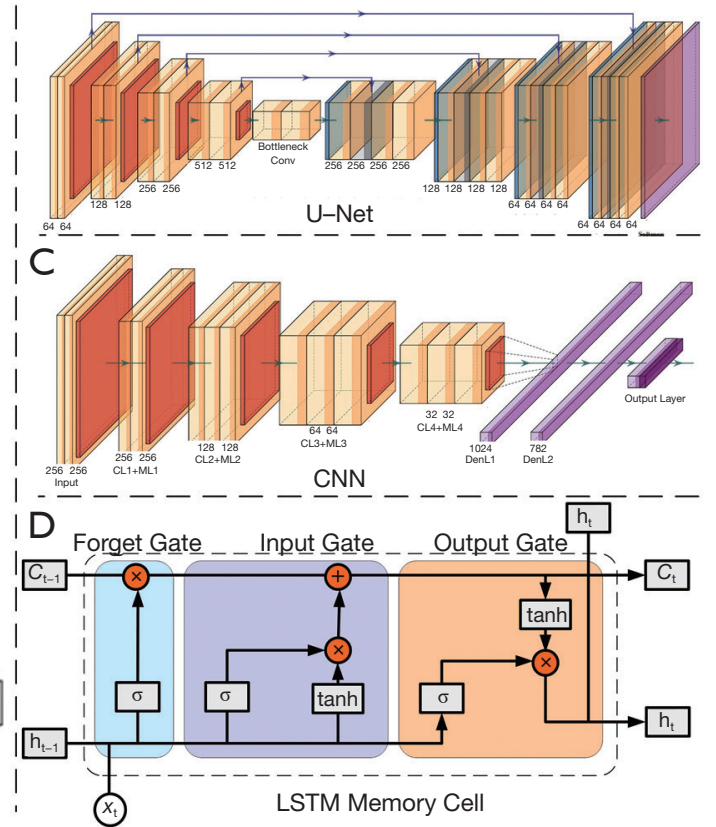

Figure 10 The DL System architectures of (A) The global architecture, (B) U-Net, (C) CNN, and (D) LSTM memory cell (courtesy of AtheroPoint, Roseville, CA, USA). DL, deep learning; CNN, convolutional neural network; LSTM, long short-term memory.
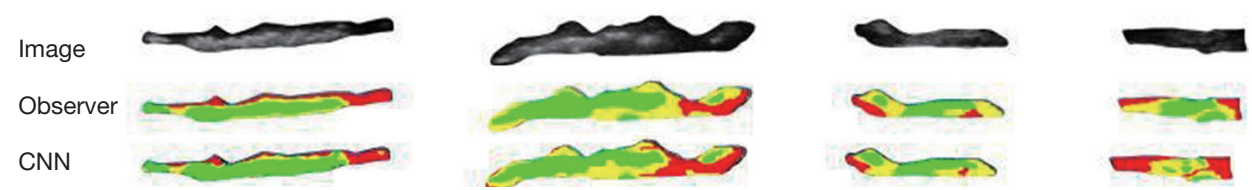

Figure 11 A visual example of DL-based CNN classification results shown in color representing different components of plaque [source: (169), Permitted for reproduction]. CNN, convolutional neural network; DL, deep learning.

architecture of U-Net. The encoders compress the image, and the decoders do the opposite. RNN is typically used when time series or historical clinical data are essential (164). Meanwhile, LSTM is useful when the input data is present within the time sequence (165). Finally, RCNN and Mask R-CNN are used for segmentation (166).

Figure $10 C$ represents a conventional convolution neural network with a convolution layer followed by a maxpooling layer. Figure 10D depicts an LSTM recurrent neural network. It consists of three gates. The forget gate removes information that is no longer useful in the cell state, the input gate provides additional information that is required for the cell state. The output gate extracts all useful information from the current cell state.

Demirer et al. (167) developed a GUI-based toolbox using a deep neural network (DNN) for 2-D/3-D segmentation and classification and integration with third-party scripting languages. The researchers used this tool to annotate 1,843 arteries and 294 coronary CTA atherosclerotic plaques in 23 days. This GUI used NoSQL as its database, while TensoFlows was used for DNN. In other work, Lee et al. (168) developed a DL model and used it to classify 6,556-lumen images of OCT scans using CNN. The authors achieved sensitivity and specificity rates of $84.8 \%$ and $91.4 \%$ (fibrolipidic) and $97.8 \%$ and $95.7 \%$ (fibrocalcific).

Lekadir et al. (169) proposed a CNN model that classifies plaque components into lipid core, fibrous cap, and calcified tissue. The authors did this by extracting 90,000 patches from 50 in vivo CUS. The authors achieved a correlation coefficient of 0.90 between automatic measurement and expert measurement (see Figure 11 for a visual representation of CNN classification). Skandha 


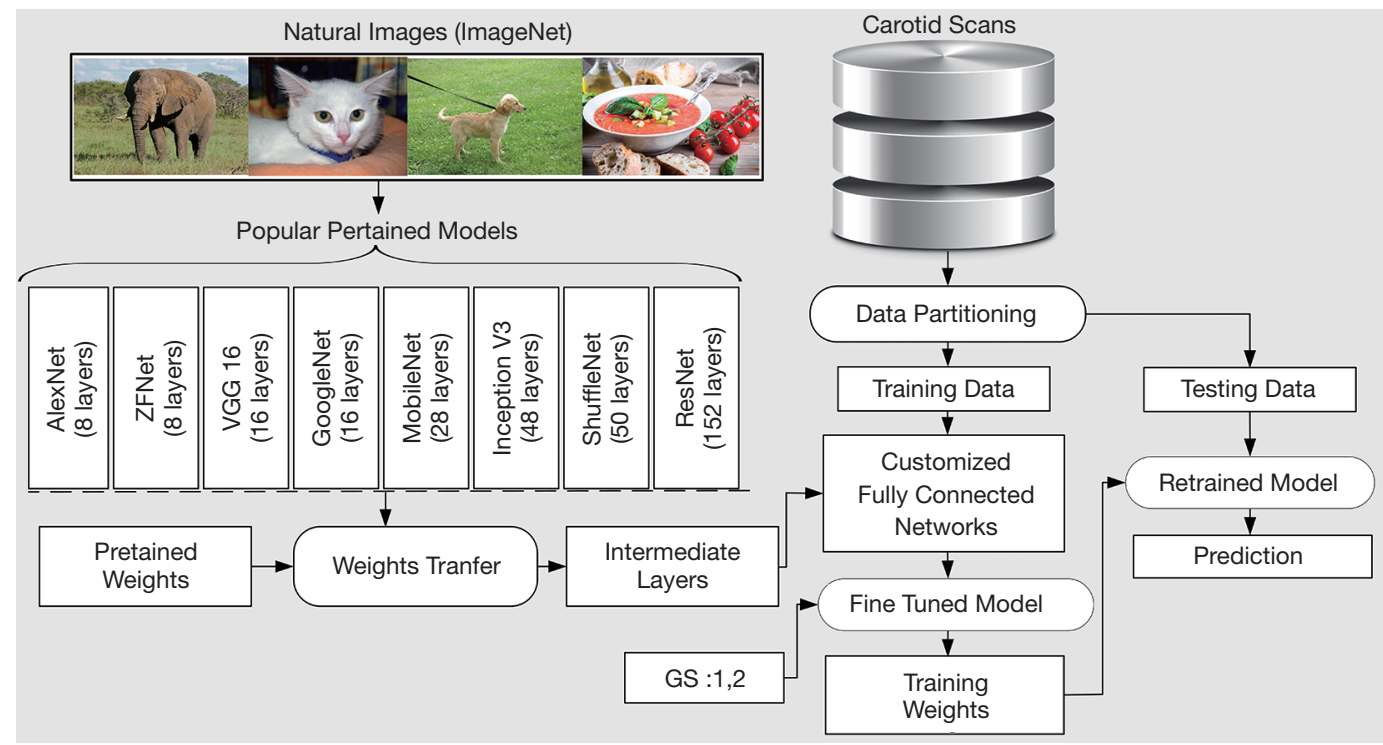

Figure 12 The global architecture of the TL models (courtesy of AtheroPoint, Roseville, CA, USA). TL, transfer learning.

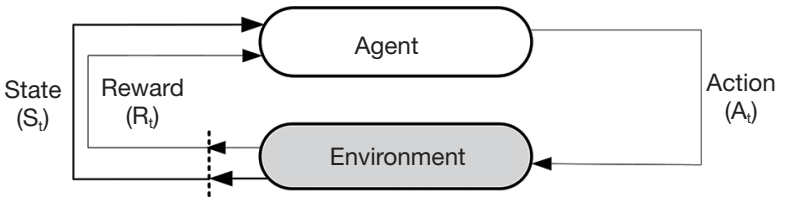

Figure 13 Reinforcement learning architecture (courtesy of AtheroPoint, Roseville, CA, USA).

et al. (55) categorized 346 CUS scans as either symptomatic or asymptomatic using optimized deep CNN. They also characterized plaque higher features using mean feature strength (MFS) and bispectrum. The authors achieved an accuracy of $95.66 \%$ and an AUC of $0.956(\mathrm{P}<0.0001)$. Research on the use of DL in TCCCA is still emerging (170); other researchers have used DL to investigate the chest $(29,171)$, coronary $(172)$, liver $(28)$, IMT wall $(173,174)$ of patients, as well as lumen characterization (26) and carotid risk measurement in diabetic patients $(30,56)$ and Rheumatoid arthritis (175) in arthritic patients in particular.

\section{Transfer learning model in MR, CT, and the US}

DL is a rapidly growing technology, but it has a minor drawback (i.e., the high-performance hardware requirements for and availability. This issue is resolved by the transfer learning technique, i.e., by using a DL model that has been pre-trained for natural images. The weights are transferred to the current target model by the researcher. Then, the researcher can train the FCN for target label prediction (27), thus avoiding the problem associated with the availability of high-quality computational devices. The global architecture of the TL system is shown in Figure 12.

\section{Reinforcement learning (RL)}

$\mathrm{RL}$ is a unique $\mathrm{AI}$ technique that enables a model to learn complex patterns using trial-and-error methods based on its own experience (176). Figure 13 presents an architectural view of reinforcement learning.

\section{AI similarities and differences between $M R, C T$, and $U S$}

While we have seen that AI models independently operate for TCCCA using MR, CT, and US, but it is necessary to study the differences and similarities. Table 2 shows the difference between MR, CT, and US for TCCCA using AI. We distinguish the three modalities using the three prominent AI models, namely, ML, DL, and TL. These AI models are divided into different blocks, such as segmentation, feature extraction, classification, and performance metrics. The corresponding references are also attached for ready reference. Table 3 shows the similarities between the modalities when it comes to AI models. The differences and similarities are selfdescribed and explanatory. We, therefore, will not elaborate in detail here. 
Table 2 Difference between MR, CT, and US for TCCCA using AI

\begin{tabular}{|c|c|c|c|}
\hline & MRI & CT & US 2-D/3-D \\
\hline \multirow{4}{*}{ ML } & $\begin{array}{l}\text { Segmentation: Bayes } \\
\text { Clustering, Structural } \\
\text { Support Vector Machines, } \\
\text { Manual, SNAP, 3-D } \\
\text { Hybrid Segmentation, 3-D } \\
\text { MARGE }\end{array}$ & Segmentation: automatic & $\begin{array}{l}\text { Segmentation: Manual, Simple Linear Iterative clustering, RealAdaboost, } \\
\text { Bayesian }\end{array}$ \\
\hline & $\begin{array}{l}\text { Extracted Features: } \\
\text { Surface disruption } \\
\text { features, SIFT, } \\
\text { Morphological, Intensity } \\
\text { Features of IR, Intensity } \\
\text { features of reference } \\
\text { acquisition (REF), } \\
\text { TOF-MRA and BBMRI, } \\
\text { histological }\end{array}$ & $\begin{array}{l}\text { Extracted Features: Texture } \\
\text { features and relative } \\
\text { position of pixels, LBP and } \\
\text { wavelet transform, Texture } \\
\text { features }\end{array}$ & $\begin{array}{l}\text { Extracted Features: Multiresolution Features, Bi-dimensional empirical } \\
\text { mode decomposition, and entropy features, texture features, automatic, } \\
\text { Rayleigh Mixture Model, Histogram, Texture, Morphological, Monogenic, } \\
\text { Wavelet energies, co-occurrence matrix, } 1^{\text {st }} \text { order statistics, Multilevel } \\
\text { binary morphological, second-order statistics spatial gray level dependence } \\
\text { matrices, ACRS Clinical, discrete wavelet and higher-order spectra, 2-D } \\
\text { DWT, Degree of stenosis, DWT with Averaging. Envelop Radio-Frequency, } \\
\text { statistical features, fractal dimension, laws texture energy, Fourier power } \\
\text { spectrum, Spatial Based Plaque Feature, 3-D Plaque Feature Extraction, } \\
\text { Neighbourhood Gray Tone Difference Matrix, Quadratic Programming } \\
\text { Feature Selection, Minimal Redundancy Maximal Relevance, Mutual } \\
\text { Information Quotient, Spectral Conditional Mutual Information, Cramer's V } \\
\text { test, neighborhood gray-tone difference matrix, 3-D Fractal Dimension }\end{array}$ \\
\hline & $\begin{array}{l}\text { Classification: RF, } t \text {-test } \\
\text { and logistic regression, } \\
\text { SSVM, NB, SVM, RF, } \\
\text { GBDT, ANN, KNN, SVM, } \\
\text { DT, }\end{array}$ & $\begin{array}{l}\text { Classification: RF, SVM } \\
\text { RBF, DT, NB, LR, NN }\end{array}$ & $\begin{array}{l}\text { Classification: SVM and Probabilistic Neural Network, SVM RBF, SVM } \\
\text { Polynomial, linear, LibSVM, DT, AtheroRisk, Adaboost, self-organizing map, } \\
\text { KNN, ANN, 3-D Blanket, SVM with 3-DUS }\end{array}$ \\
\hline & $\begin{array}{l}\text { Performance metrics: AUC } \\
\text { Ranges: } 0.95 ; \text { ACC ranges } \\
\text { (\%): } 87,88,76,87.5,90 \\
\text { Misclassification Rate (\%): } \\
9.6\end{array}$ & $\begin{array}{l}\text { Performance Metrics: ACC } \\
\text { Ranges (\%): } 83.1,88,69\end{array}$ & $\begin{array}{l}\text { Performance Metrics: AUC Ranges: } 0.649,0.732,0.905 ; \text { ACC Ranges (\%): } \\
85,91.43,82.4,83.5,73.7,77.18,76,91.7,83.7,90.66,83.7,99.2,73.1 \\
73.72,68.8,69.3,81.82,80.38,81\end{array}$ \\
\hline \multirow{3}{*}{$\mathrm{DL}$} & $\begin{array}{l}\text { Extracted Features: } \\
\text { Morphological features }\end{array}$ & $\begin{array}{l}\text { Extracted Features: } \\
\text { Automatic }\end{array}$ & Extracted Features: Automatic \\
\hline & Classification: DeepMAD & $\begin{array}{l}\text { Classification: } \\
\text { DeepSymNet, Faster } \\
\text { R-CNN }\end{array}$ & Classification: Optimized CNN, Dynamic CNN \\
\hline & $\begin{array}{l}\text { Performance Metrics: ACC } \\
\text { Ranges (\%): } \\
99.1,92.6,89.16\end{array}$ & $\begin{array}{l}\text { Performance Metrics: AUC } \\
\text { Ranges: } 0.88, \\
\text { ACC Ranges (\%): } 83\end{array}$ & $\begin{array}{l}\text { Performance Metrics: ACC Ranges (\%): 95.66, Dice Coefficient Ranges: } \\
96.6,84\end{array}$ \\
\hline
\end{tabular}

Table 2 (continued) 
Table 2 (continued)

\begin{tabular}{lll}
\hline MRI & CT & US 2-D/3-D \\
\hline TL & $(189)$ & $(55)$ \\
Segmentation: Automatic & $<\mathrm{NF}>$ & Segmentation: Manual \\
$\begin{array}{l}\text { Extracted Features: } 23 \\
\text { different morphological }\end{array}$ & $<\mathrm{NF}>$ & Extracted Features: Automatic \\
$\begin{array}{l}\text { Classification: Linear } \\
\text { Discriminate Classification }\end{array}$ & $<\mathrm{NF}>$ & Classification: VGG16 \\
$\begin{array}{l}\text { Performance Metrics: } \\
\text { ACC Ranges (\%):90 }\end{array}$ & $<\mathrm{NF}>$ & Performance Metrics: ACC Ranges (\%):83.33 \\
\hline
\end{tabular}

MR, magnetic resonance; CT, computed tomography; US, ultrasound; TCCCA, tissue characterization and classification of the carotid artery; Al, artificial intelligence; IR, inversion recovery; BBMRI, black blood MRI; LBP, local binary patterns.

Table 3 The similarity between MR, CT, and US for TCCCA using AI

- All the segmentation and classification was attempted on 2-D slices without considering the 3-D spatial information

- All the modalities have implemented segmentation of the wall as its first step

- The centreline algorithm was adapted to extract the orthogonal slices to the blood flow for all three modalities

- All the modalities have attempted tissue characterization

- ML has been attempted on all the three modalities for TCCCA

- $\quad$ SVM, RF, and DT are the common ML classifiers adapted by three modalities

- DL has been attempted on all the three modalities for TCCCA

- U-Net is the most common architecture used in the DL framework for all three modalities.

- $\quad \mathrm{CNN}$ is the most popular architecture tried for all modalities

- $T L$ is the least adopted among all the architectures

- Accuracy and AUC are the common performance metrics for all the three modalities

MR, magnetic resonance; CT, computed tomography; US, ultrasound; TCCCA, tissue characterization and classification of the carotid artery; Al, artificial intelligence.

\section{Carotid artery disease and its link to coronary artery disease}

Since CVD covers both stroke and myocardial infarction, it is therefore important to look at the AI trends for CVD prediction using carotid ultrasound. Accurate cardiovascular event predictions are necessary since it saves the lives of patients. This section shows how we can use the plaque deposited in the carotid artery to predict cardiovascular events (so-called myocardial infarction). This not only reduces the costs of invasive coronary artery disease screening but also offers a non-invasive imaging option for predicting cardiovascular events early. This subsection is divided into three parts. Part A presents the genetic makeup of atherosclerosis disease in the carotid artery $v s$. the coronary artery. This is accompanied by the visualization of plaque using non-invasive carotid imaging. Part B presents scientific evidence gathered from several studies that have linked plaque in the carotid artery with coronary stenosis. Finally, part $\mathrm{C}$ discusses the use of novel multiclass AI techniques for predicting coronary artery risk based on carotid plaque levels detected via ultrasound.

\section{A: atherosclerotic components and genetic makeup in carotid and coronary arteries}

Atherosclerosis is a systemic condition. It has been wellestablished that plaque seen in the coronary artery has the same set of components as plaque in the carotid artery, especially in the bulb or bifurcation region (190). Several studies have cited the presence of plaque components such 
A

B

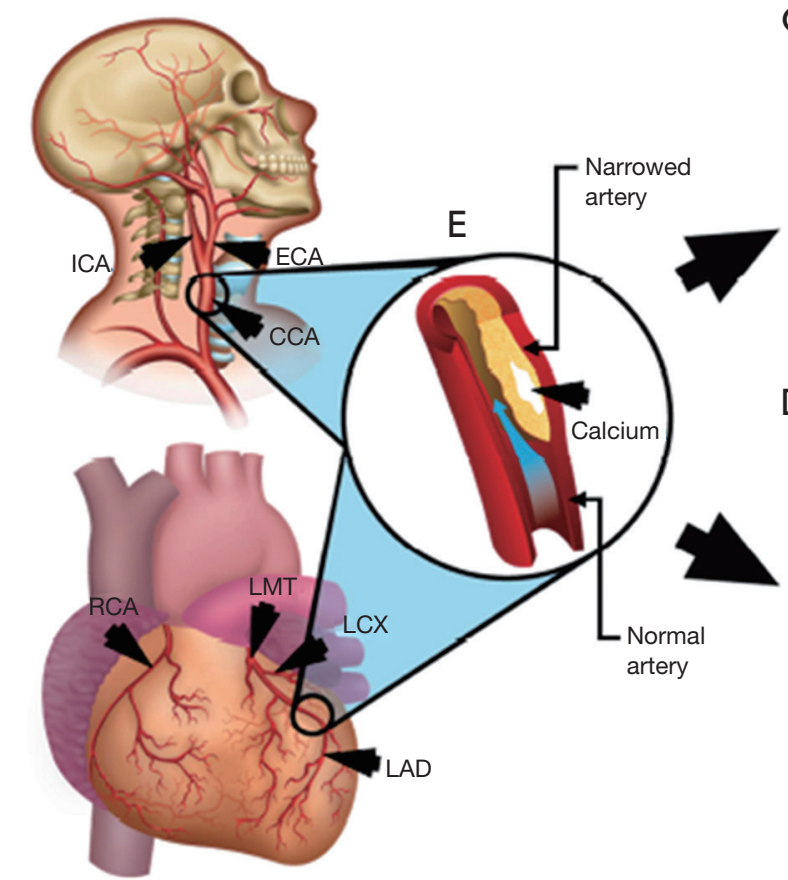

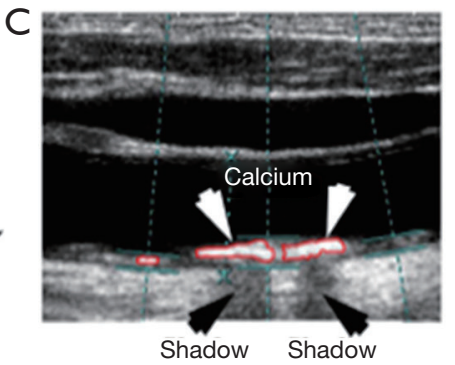

D

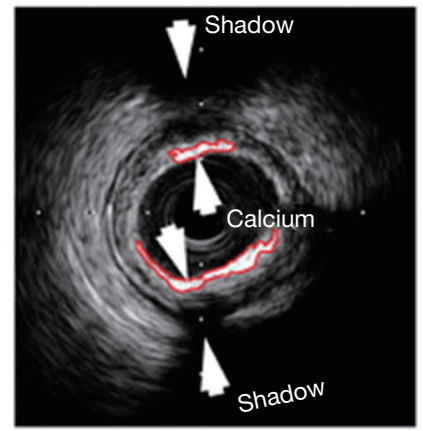

Figure 14 Carotid and coronary arteries representation of (A) and (B) visuals, (C) and (D) ultrasound scans of carotid and coronary arteries with calcium components, and $(\mathrm{E})$ a cross-section of the plaque representing blood flow disruption due to calcium deposition along artery walls [courtesy of AtheroPoint ${ }^{\mathrm{TM}}$, CA, USA, source: (140), permitted for reproduction].

as lipid, fibrosis, fibrin, and calcium in both the arteries (I) via IVUS or CT (in the coronary artery) (191) and (II) via MR/CT/BUS (in the carotid artery) $(5,106,125,192)$. An example of the link between the plaque burden in the carotid and coronary arteries can be seen in Figure 14. This similarity of plaque composition has been validated by several pathology studies (193-195).

\section{B: the relationship between carotid and coronary artery disease}

Several studies have verified that carotid artery plaque measurements are directly related to coronary artery disease and cardiovascular disease risk (5,196-198). Clinical trials (199) have revealed that carotid IMT is a useful surrogate marker for coronary vascular disease. Further, in (196), the authors used cIMT for carotid and coronary arteries while using an ultrasound framework. The authors in (200) showed maximum plaque height $(\mathrm{MPH})$ as a measure to evaluate the coronary artery disease risk. Further, authors in (201-203) showed the carotid bulb's use to assess coronary artery disease risk. Table 4 depicts the correlation between carotid-to-coronary and clearly establishes the validity of estimating the risk of CVD, MI, or CVE based on carotid plaque levels.

\section{C: AI framework for prediction of coronary artery disease based on the carotid artery}

Several studies have been carried out to predict carotid and coronary artery disease risk in the ML framework $(151,212,213)$. Recently, an ML-based strategy was developed for determining the risk of coronary artery disease using the carotid artery as a gold standard $(139,214,215)$. ML was also applied to identify coronary artery disease patients using the greyscale features of left ventricle ultrasound scans (216).

In recent research, intravascular neovascularization (IPN) (206) was used to detect plaque in carotid arteries. The observations were then used to predict coronary artery disease based on univariate logistic regression. The authors in (217) extended the role of IPN, along with carotid B-mode ultrasound (CBUS) and office-based biomarkers (OBBM), in the ML framework to predict coronary artery disease. The ML method provided superior coronary artery disease risk assessments, as it was able to differentiate between the non-linearity between risk factors (predictors) and the gold 
Table 4 Studies are showing the relationship between carotid plaque and coronary arteries

\begin{tabular}{|c|c|c|c|c|}
\hline SN & Ref & Relationship between carotid vs. coronary arteries & Technique & Performance metrics \\
\hline 2 & & & & Specificity: $80 \%$ \\
\hline 3 & $(206)$ & $\begin{array}{l}\text { Effect of carotid plaque vulnerability on coronary artery } \\
\text { disease using IPN }\end{array}$ & The Kaplan-Meier analysis & IPN score $>1.25, P=0.004$ \\
\hline 4 & $(207)$ & $\begin{array}{l}\text { Atherosclerosis risk factor and calcium score of } \\
\text { middle-age men in the femoral and carotid arteries } \\
\text { improves the CVD risk prediction in coronary }\end{array}$ & $\begin{array}{l}\text { Femoral odds ratio, carotid odds } \\
\text { ration }\end{array}$ & AUC: 0.665 to 0.719 \\
\hline 5 & $(208)$ & $\begin{array}{l}\text { Al and IVUS based framework for measuring the } \\
\text { coronary risk assessment from the cIMT and validated } \\
\text { relation between two arteries }\end{array}$ & $\begin{array}{l}\text { SVM with RBF, poly order } 1,2,3 \text {, } \\
\text { and linear }\end{array}$ & $\begin{array}{l}\text { ACC (\%): } 94.95 \text { AUC: } \\
0.95\end{array}$ \\
\hline
\end{tabular}

7 (201) Automated 3-D ultrasound-based carotid plaque quantification is a useful screening tool for $C A D$

Stacked-contour method \& 16-segment model
Sensitivity (\%) 98.0, 93.9
8

(200) An increase of cIMT thickness and plaque measurements are indicative of the presence of epicardial coronary stenosis

9

(211) Effect of calcium score on coronary artery over carotid wall plaque
Mean far distal carotid intima-media thickness, maximum plaque height, total plaque area

Kaplan-Meier analysis

Cox proportional hazards models, C-statistics
An optimal threshold value of cIMT thickness of $0.82 \mathrm{~mm}$

Plaque height of $1.54 \mathrm{~mm}$

Plaque area: $25.6 \mathrm{~mm}^{2}$

Survival analysis at six years by Kaplan-Meier estimates was 95.6

Coronary vascular disease Hazard ratio: 1.78, $\mathrm{P}<0.001$ Carotid vascular disease Hazard ratio 2.09, $\mathrm{P}<0.001$

Plaque area

$\mathrm{P}<0.05$
(203) Left main coronary artery atherosclerosis is related to maximum common cIMT by measuring with carotid ultrasonography
(202) Contralateral carotid artery stenosis and high-intensity carotid plaque on T1 weighted MRI predicts the CoVD

CoVD, coronary vascular disease; IPN, intraplaque neovascularization; CAD, coronary artery disease; CEA, carotid endarterectomy.

standard (CVE). These strategies can also be applied to carotid plaque data while using coronary angiography score (CAS) as the gold standard. Such an automated system is likely to become a prominent $\mathrm{CAD}$ detection system in the future. Further, the team (55) published the DL strategy for stroke risk prediction using the gold standard as carotid risk labels, which can be extended to coronary risk prediction if the gold standard was taken from the coronary artery. 

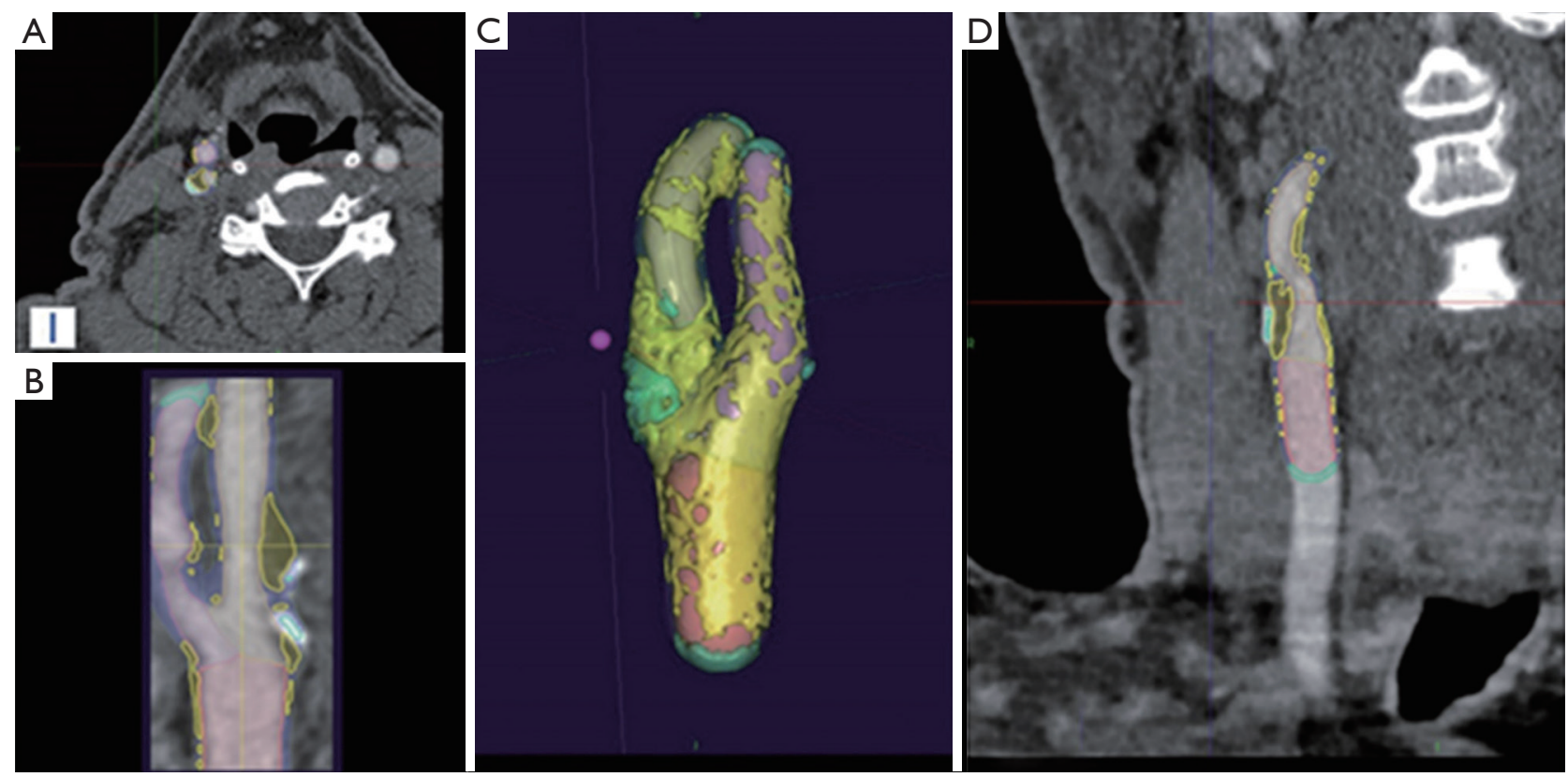

Figure 15 Visual 3-D MRI cross-sectional representations of severe stenosis in the right internal artery: (A) axial, (B) sagittal, and (D) coronal. (C) represents the 3-D segmentation of lumen, vessel wall, and plaque components, Green: calcification, Yellow: plaque components, and Blue: background matrix [courtesy of AtheroPoint ${ }^{\mathrm{TM}}$, CA, USA, source: (227), Permitted for reproduction]. MRI, magnetic resonance imaging.

\section{Critical discussion}

\section{Image acquisition, normalization, and automated segmentation/manual delineation}

In CT, the Hounsfield Unit (HU) value plays an important role in carotid plaque characterization (218). Saba et al. (219) recently studied the correlation between the carotid computed tomography (CT) Hounsfield unit (HU)-based plaque attenuation values measured using dual-energy CT (DECT) scanner and brain leukoaraiosis (LA). The study showed an association between HU attenuation of the carotid artery plaques and the LA volume at low keV energy levels.

In the US, the transducer angle (angle of insonation) plays an important role in ultrasound imaging. The angle of insonation is equal to the angle of the ultrasound beam relative to the tissue of interest (220). Maximizing the amplitude of the target's echo through the transducer provides optimal imaging at specular reflector (a perpendicular incident beam causes perpendicular reflection) per, and amplitude depends on the orientation, size, and its surface characteristics. Thus, obtaining the specular reflector is essential for imaging in an ultrasound.
Classification accuracy depends on the normalization methods used as well as the intensity levels of the images. Normalization is the first step after image acquisition; it is a statistical process that compares inappropriate scaling. In general, MRI, CT, and US imaging undergo normalization to generate more interpretable final images.

Plaque delineation in the existing works was done manually $(52,61,62,93,221,222)$ using a mouse and video card. Medical practitioners selected the plaque region in the full scan and created a mask over it to cut the plaque of the ROI. This method is error-prone and requires practitioners skilled in delineation. Using advanced AI, like RCNN and Mask RCNN, may help in the automatic segmentation of plaque (223).

\section{3-D imaging and carotid geometry}

Three-dimensional medical imaging is required in order to understand an organ's spatial information. In 3-D MRI, all the slices of 2-D MRI are joined from top to bottom using advanced computational techniques (224,225). Similarly, in CT scans, a series of 2-D scans are mapped to create a 3-D visualization. In the US as well, 2-D US scans were mapped to a $3-\mathrm{D}$ visualization; however, this visualization carries an 

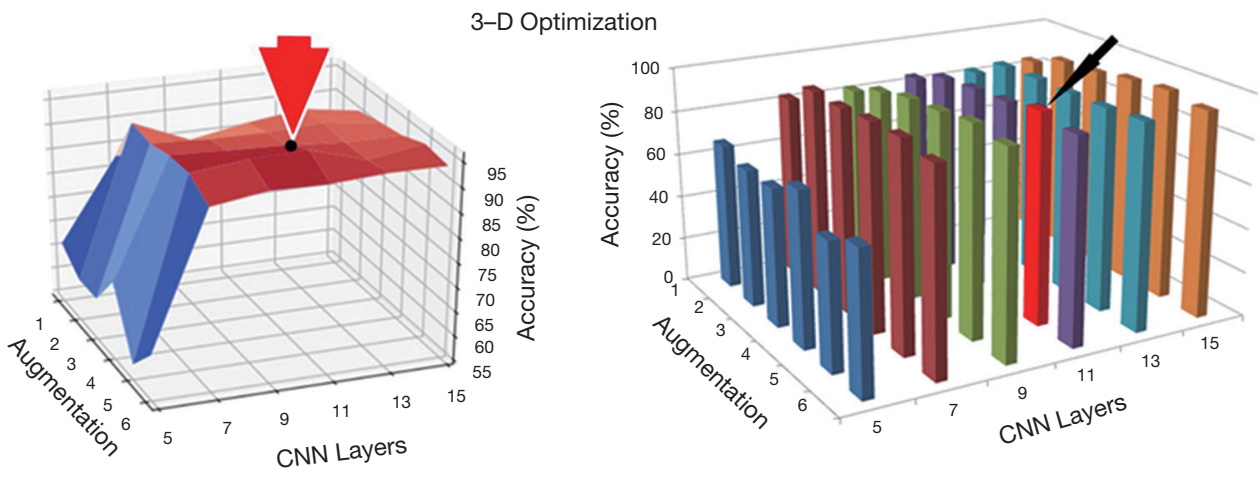

Figure 16 Left: 3-D optimization of DCNN showing CNN layers vs. augmentation vs. accuracy. Right: 3-D bar chart of the optimized DCNN (11 CNN layers and 5-fold augmentation). [courtesy of AtheroPoint ${ }^{\mathrm{TM}}$, CA, USA, source: (55), Permitted for reproduction]. DCNN, deep convolutional neural network; CNN, convolutional neural network.

A

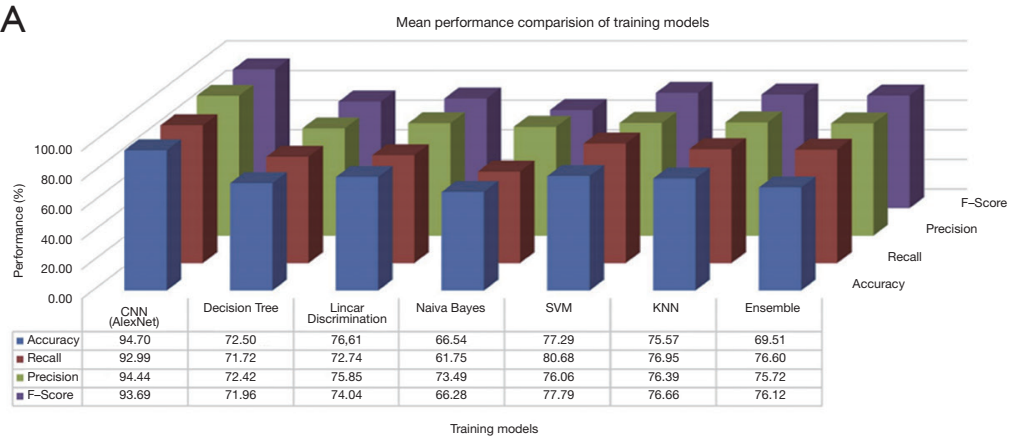

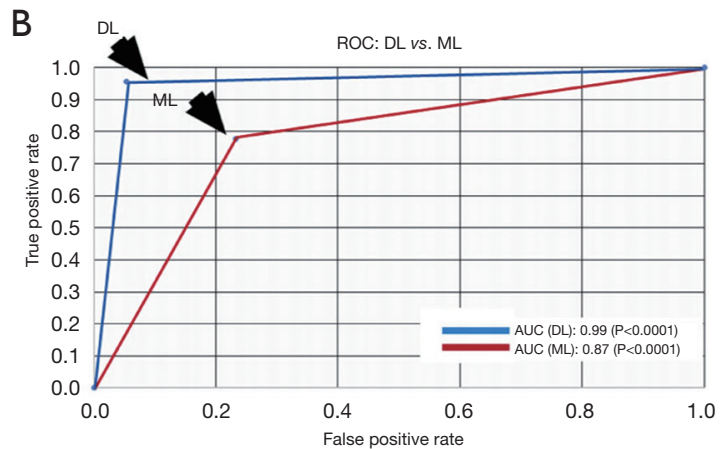

Figure 17 Example showing (A) performance of ML vs. DL training models (classifiers) in multiclass framework utilizing T2-weighted MRI brain scans, and (B) corresponding ROC plots of ML vs. DL in the multiclass framework [courtesy of AtheroPoint ${ }^{\mathrm{TM}}$, CA, USA, source: (229), Permitted for reproduction]. ML, machine learning; DL, deep learning; MRI, magnetic resonance imaging.

in-depth analysis of the tissue that actually reduces operator dependence (184). 3-D imaging offers a more accurate diagnosis for advanced atherosclerosis (226). See Figure 15 for 3-D MRI. Saba et al. (228) showed that the index of asymmetry in the carotid artery wall thickness (CAWT) was used as a further parameter to stratify the risk of symptoms related to the carotid artery.

\section{3-D Optimization}

AI techniques must be optimized to achieve more accurate predictions and risk assessments. 3-D optimization is a novel technique that varies with the hyperparameters of the model; variation may exist in the number of features, the layers, the nodes, the data size, or the optimal point at which the AI model will give the best results (55). Figure 16 depicts the 3-D optimization of CNN (courtesy of Atheropoint ${ }^{\mathrm{TM}}$ ).

\section{Multi-class classification}

AI studies on plaque classification have focused on binary classification $(40,52,55,61,62,93,94)$ i.e., symptomatic (unstable) vs. asymptomatic (stable). However, in reality, this classification is more of a spectrum, including symptomatic, moderate symptomatic, weakly symptomatic, and asymptomatic. It is thus a multiclass classification problem. Dealing with a problem of this nature demands a great deal of data analysis to determine the misclassification ratio (100). Our team considered the problem of carotid plaque to be one of multiclass classification. Tandel et al. (229) performed a multiclass classification (from three classes to six classes) when exploring the different stages of brain tumors in T2 MRI brain scans using seven AI techniques. Figure 17 represents the ROC curves and performance metrics of the multiclass classification using DL and ML (229). 


\section{Hardware analysis}

Advanced AI applications require sophisticated hardware (GPUs). We worked on two supercomputers designed for deep learning applications-one at Banaras Hindu University (BHU) (Param Shivay), in Varanasi, and the other at Bennett University (BU), in New Delhi. BHU's supercomputer is a grid computer, while BU's is a standalone machine developed by Nvidia. Training a DL model at BHU took 9 seconds per epoch, whereas, at BU, that time increased to 18 seconds; the grid computation structure at BHU required less training time. BHU and BU have the same Nvidia GPUs (i.e., Nvidia DGX V100). However, every job at BU is assigned to a single GPU because it is a standalone system. Meanwhile, the system at BHU has 233 nodes; each job is assigned to $40 \mathrm{CPU}$ nodes and 2 GPU nodes, meaning jobs can run concurrently.

\section{Interface, validation, and extensions}

Interfacing AI techniques with real-time data through a graphical user interface has the potential to lighten the radiologist's burden of image data curation, annotations, segmentation, and risk prediction (167). AI tools such as qQuant, ImageJ, and RADSpa can help radiologists access and characterize malignancy in tissues.

The probability scores generated by AI models can be validated using both ground truth values and neovascularization (206). In the latter technique, a score is assigned to the plaque depending on the microbubbles present; based on this, an inter-plaque neovascularization score is calculated and used to predict cardiovascular events.

Similar work can be done on the coronary, aorta, or peripheral arteries and neural organs; numerous AI applications exist in the area of tissue classification and characterization. Further comparison of carotid plaques generated in different geological locations would also yield more sophisticated results.

\section{Conclusion}

This review presented the AI-based models for multimodality TCCCA. A search strategy was adopted using a standardized protocol that adapted inclusion and exclusion criteria. The order of AI models in multimodality TCCCA was US>CT >MRI $>$ IVUS $>$ PET. Over $95 \%$ of studies adopted 2-D imaging during the AI model design.
Typically, the system consists of segmentation, followed by classification. Several studies are leaning towards automated segmentation of carotid plaque, unlike semi-automated. This study classified AI models into five categories: manual, statistical, machine learning, deep learning, and transfer learning. The order of the number of AI models implemented in TCCCA was ML $>\mathrm{DL}>\mathrm{TL}$. The ML was dominated in the order of $\mathrm{SVM}>k-\mathrm{NN}>\mathrm{ANN}$, where the most prominent feature extracted was the texture. In DL, $\mathrm{CNN}$ architecture was most adopted for classification and U-Net for segmentation. VGG16 was most popular in TL. Our observation showed a low number of validations were conducted when using AI models. One reason could be the lack of publicly available databases. It is expected that AI models will dominate the TCCCA industry for the next decade.

\section{Acknowledgments}

Funding: None.

\section{Footnote}

Provenance and Peer Review: This article was commissioned by the Guest Editor (Kosmas I. Paraskevas) for the series "Carotid Artery Stenosis and Stroke - Prevention and Treatment Part II" published in Annals of Translational Medicine. The article has undergone external peer review.

Conflicts of Interest: All authors have completed the ICMJE uniform disclosure form (available at http://dx.doi. org/10.21037/atm-20-7676). The series "Carotid Artery Stenosis and Stroke - Prevention and Treatment Part II" was commissioned by the editorial office without any funding or sponsorship. The authors have no other conflicts of interest to declare.

Ethical Statement: The authors are accountable for all aspects of the work in ensuring that questions related to the accuracy or integrity of any part of the work are appropriately investigated and resolved.

Open Access Statement: This is an Open Access article distributed in accordance with the Creative Commons Attribution-NonCommercial-NoDerivs 4.0 International License (CC BY-NC-ND 4.0), which permits the noncommercial replication and distribution of the article with the strict proviso that no changes or edits are made 
and the original work is properly cited (including links to both the formal publication through the relevant DOI and the license). See: https://creativecommons.org/ licenses/by-nc-nd/4.0/.

\section{References}

1. Prevention CfDCa. 2020. Available online: https:// www.cdc.gov/media/pressrel/2010/r101022.html \#\%3A \%3Atext \%3DAs\%20many\%20as\%201\%20i. Accessed 10 June 2020.

2. Mackay J, Mensah GA. The atlas of heart disease and stroke. World Health Organization; 2004.

3. Virani SS, Alonso A, Benjamin EJ, et al. Heart disease and stroke statistics-2020 update: a report from the American Heart Association. Circulation 2020;141:e139-e596.

4. Libby P. Inflammation and cardiovascular disease mechanisms. Am J Clin Nutr 2006;83:456S-60S.

5. Suri JS, Kathuria C, Molinari F. Atherosclerosis disease management. Springer Science \& Business Media; 2010.

6. Meijers WC, de Boer RA. Common risk factors for heart failure and cancer. Cardiovasc Res 2019;115:844-53.

7. Porcu M, Mannelli L, Melis M, et al. Carotid plaque imaging profiling in subjects with risk factors (diabetes and hypertension). Cardiovasc Diagn Ther 2020;10:1005.

8. Libby P, Ridker PM, Maseri A. Inflammation and atherosclerosis. Circulation 2002;105:1135-43.

9. Libby P, Simon DI. Inflammation and thrombosis: the clot thickens. Am Heart Assoc; 2001.

10. Ueda M. ATIS. Drugs 2010;70:3-8.

11. Saba L, Sanches JM, Pedro LM, et al. Multi-modality atherosclerosis imaging and diagnosis. Springer; 2014.

12. Radeva P, Suri JS. Vascular and Intravascular Imaging Trends, Analysis, and Challenges, Volume 2; Plaque characterization. vii2. 2019.

13. Watson MG, Byrne HM, Macaskill C, et al. A multiphase model of growth factor-regulated atherosclerotic cap formation. J Math Biol 2020;81:725-67.

14. Matsubara K, Ibaraki M, Shidahara M, et al. Iterative framework for image registration and partial volume correction in brain positron emission tomography. Radiol Phys Technol 2020;13:348-57.

15. Saba L, Yuan C, Hatsukami T, et al. Carotid artery wall imaging: perspective and guidelines from the ASNR Vessel Wall Imaging Study Group and expert consensus recommendations of the American Society of Neuroradiology. AJNR Am J Neuroradiol 2018;39:E9-E31.

16. Seyman EE, Bornstein N, Auriel E, et al. Assessment of carotid artery ultrasonography in the presence of an acoustic shadow artifact. BMC Neurol 2019;19:178.

17. Nicolaides A, Kakkos SK, Kyriacou E, et al. Ultrasonic plaque characterization: Results from the asymptomatic carotid stenosis and risk of stroke (ACSRS) study. Ultrasound and Carotid Bifurcation Atherosclerosis. Springer; 2011. p. 613-32.

18. Takano K, Yamashita S, Takemoto K, et al. Characterization of carotid atherosclerosis with blackblood carotid plaque imaging using variable flip-angle 3D turbo spin-echo: comparison with 2D turbo spin-echo sequences. Eur J Radiol 2012;81:e304-9.

19. Acharya UR, Mookiah MRK, Sree SV, et al. Atherosclerotic plaque tissue characterization in 2D ultrasound longitudinal carotid scans for automated classification: a paradigm for stroke risk assessment. Med Biol Eng Comput 2013;51:513-23.

20. Suri JS, Pattichis CS, Li C, et al. Plaque imaging using ultrasound, magnetic resonance and computer tomography: a review. Stud Health Technol Inform 2005;113:1-25.

21. Patel AK, Suri HS, Singh J, et al. A review on atherosclerotic biology, wall stiffness, physics of elasticity, and its ultrasound-based measurement. Curr Atheroscler Rep 2016;18:83.

22. Haussen DC, Grossberg JA, Koch S, et al. Multicenter experience with stenting for symptomatic carotid web. Interv Neurol 2018;7:413-8.

23. Seabra J, Sanches J. Ultrasound Imaging: Advances and Applications. New York: Springer; 2012.

24. Pedro LM, Sanches JM, Seabra J, et al. Asymptomatic carotid disease-a new tool for assessing neurological risk. Echocardiography 2014;31:353-61.

25. Christodoulou CI, Pattichis CS, Pantziaris M, et al. Texture-based classification of atherosclerotic carotid plaques. IEEE Trans Med Imaging 2003;22:902-12.

26. Biswas M, Kuppili V, Saba L, et al. Deep learning fully convolution network for lumen characterization in diabetic patients using carotid ultrasound: a tool for stroke risk. Med Biol Eng Comput 2019;57:543-64.

27. Biswas M, Kuppili V, Saba L, et al. State-of-the-art review on deep learning in medical imaging. Front Biosci (Landmark Ed) 2019;24:392-426.

28. Biswas M, Kuppili V, Edla DR, et al. Symtosis: A liver ultrasound tissue characterization and risk stratification in optimized deep learning paradigm. Comput Methods Programs Biomed 2018;155:165-77.

29. Kats L, Vered M, Zlotogorski-Hurvitz A, et al. Atherosclerotic carotid plaques on panoramic imaging: an 
automatic detection using deep learning with small dataset. arXiv preprint arXiv:180808093 2018.

30. Saba L, Biswas M, Suri HS, et al. Ultrasound-based carotid stenosis measurement and risk stratification in diabetic cohort: a deep learning paradigm. Cardiovasc Diagn Ther 2019;9:439.

31. Winn WB, Schmiedl UP, Reichenbach DD, et al. Detection and characterization of atherosclerotic fibrous caps with T2-weighted MR. AJNR Am J Neuroradiol 1998;19:129-34.

32. Yuan C, Mitsumori LM, Beach KW, et al. Carotid atherosclerotic plaque: noninvasive MR characterization and identification of vulnerable lesions. Radiology 2001;221:285-99.

33. Seabra JC, Sanches J, Pedro LM, et al., editors. Carotid plaque $3 \mathrm{~d}$ compound imaging and echo-morphology analysis: a bayesian approach. 2007 29th Annual International Conference of the IEEE Engineering in Medicine and Biology Society; 2007: IEEE.

34. Loizou CP, Kyriacou E. Ultrasound Asymptomatic Carotid Plaque Image Analysis for the Prediction of the Risk of Stroke. Cardiovascular Computing-Methodologies and Clinical Applications. Springer; 2019. p. 317-29.

35. Lambrou A, Papadopoulos H, Kyriacou E, et al. Evaluation of the risk of stroke with confidence predictions based on ultrasound carotid image analysis. Int J Artif Intell Tools 2012;21:1240016.

36. Kyriacou E, Vogazianos P, Christodoulou C, et al., editors. Prediction of the time period of stroke based on ultrasound image analysis of initially asymptomatic carotid plaques. 2015 37th Annual International Conference of the IEEE Engineering in Medicine and Biology Society (EMBC); 2015: IEEE.

37. Tsiaparas NN, Golemati S, Andreadis I, et al. Comparison of multiresolution features for texture classification of carotid atherosclerosis from B-mode ultrasound. IEEE Trans Inf Technol Biomed 2011;15:130-7.

38. Tsiaparas N, Golemati S, Andreadis I, et al. Assessment of carotid atherosclerosis from B-mode ultrasound images using directional multiscale texture features. Meas Sci Technol 2012;23:114004.

39. Gastounioti A, Makrodimitris S, Golemati S, et al. A novel computerized tool to stratify risk in carotid atherosclerosis using kinematic features of the arterial wall. IEEE J Biomed Health Inform 2015;19:1137-45.

40. Acharya UR, Sree SV, Krishnan MMR, et al. Atherosclerotic risk stratification strategy for carotid arteries using texture-based features. Ultrasound Med Biol
2012;38:899-915.

41. Hernandez-Vila E, Strickman NE, Skolkin M, et al. Carotid stenting for post-endarterectomy restenosis and radiation-induced occlusive disease. Tex Heart Inst J 2000;27:159.

42. Khedr H, Eweda A, Hamza M, et al. Carotid endarterectomy versus carotid artery stenting without protection devices for the management of carotid artery stenosis. The Egyptian Journal of Surgery 2016;35:225.

43. Saba L, Suri JS. Multi-detector CT imaging: abdomen, pelvis, and CAD applications. CRC Press; 2013.

44. El-Baz AS, Saba L, Suri J. Abdomen and thoracic imaging. Springer; 2014.

45. El-Baz A, Suri JS. Neurological Disorders and Imaging Physics, Volume 3; Application to autism spectrum disorders and Alzheimer's. ndi3 2019.

46. Sinha GR, Suri JS. Cognitive Informatics, Computer Modelling, and Cognitive Science: Volume 1: Theory, Case Studies, and Applications. Academic Press; 2020.

47. Acharya UR, Sree SV, Ribeiro R, et al. Data mining framework for fatty liver disease classification in ultrasound: a hybrid feature extraction paradigm. Med Phys 2012;39:4255-64.

48. Saba L, Dey N, Ashour AS, et al. Automated stratification of liver disease in ultrasound: an online accurate feature classification paradigm. Comput Methods Programs Biomed 2016;130:118-34.

49. Molinari F, Mantovani A, Deandrea M, et al. Characterization of single thyroid nodules by contrastenhanced 3-D ultrasound. Ultrasound Med Biol 2010;36:1616-25.

50. Shrivastava VK, Londhe ND, Sonawane RS, et al. Computer-aided diagnosis of psoriasis skin images with HOS, texture and color features: a first comparative study of its kind. Comput Methods Programs Biomed 2016;126:98-109.

51. Suri JS. Imaging based symptomatic classification and cardiovascular stroke risk score estimation. Google Patents; 2011.

52. Acharya UR, Faust O, Sree SV, et al. An accurate and generalized approach to plaque characterization in 346 carotid ultrasound scans. IEEE Trans Instrum Meas 2011;61:1045-53.

53. Martis RJ, Acharya UR, Prasad H, et al. Application of higher order statistics for atrial arrhythmia classification. Biomed Signal Process Control 2013;8:888-900.

54. Saba L, Biswas M, Kuppili V, et al. The present and future of deep learning in radiology. Eur J Radiol 2019;114:14-24. 
55. Skandha SS, Gupta SK, Saba L, et al. 3-D optimized classification and characterization artificial intelligence paradigm for cardiovascular/stroke risk stratification using carotid ultrasound-based delineated plaque: Atheromatic ${ }^{\mathrm{TM}}$ 2.0. Comput Biol Med 2020;125:103958.

56. Biswas M, Kuppili V, Araki T, et al. Deep learning strategy for accurate carotid intima-media thickness measurement: an ultrasound study on Japanese diabetic cohort. Comput Biol Med 2018;98:100-17.

57. S Tandel G, Biswas M, G Kakde O, et al. A Review on a Deep Learning Perspective in Brain Cancer Classification. Cancers (Basel) 2019;11:111.

58. Kuppili V, Biswas M, Sreekumar A, et al. Extreme Learning Machine Framework for Risk Stratification of Fatty Liver Disease Using Ultrasound Tissue Characterization. J Med Syst 2017;41:152.

59. Newby AC, Zaltsman AB. Fibrous cap formation or destruction - the critical importance of vascular smooth muscle cell proliferation, migration and matrix formation. Cardiovasc Res 1999;41:345-60.

60. Davis LA, Stewart SE, Carsten III CG, et al. Characterization of fracture behavior of human atherosclerotic fibrous caps using a miniature single edge notched tensile test. Acta Biomater 2016;43:101-11.

61. Acharya RU, Faust O, Alvin APC, et al. Symptomatic vs. asymptomatic plaque classification in carotid ultrasound. J Med Syst 2012;36:1861-71.

62. Acharya UR, Faust O, Sree SV, et al., editors. Atheromatic ${ }^{\mathrm{TM}}$ : Symptomatic vs. asymptomatic classification of carotid ultrasound plaque using a combination of HOS, DWT \& texture. 2011 Annual International Conference of the IEEE Engineering in Medicine and Biology Society; 2011: IEEE.

63. Acharya UR, Sree SV, Mookiah MR, et al. Computed tomography carotid wall plaque characterization using a combination of discrete wavelet transform and texture features: A pilot study. Proc Inst Mech Eng H 2013;227:643-54.

64. Bentzon JF, Otsuka F, Virmani R, et al. Mechanisms of plaque formation and rupture. Circ Res 2014;114:1852-66.

65. Hansen L, Taylor WR. Is increased arterial stiffness a cause or consequence of atherosclerosis? Atherosclerosis 2016;249:226-7.

66. Stoka KV, Maedeker JA, Bennett L, et al. Effects of increased arterial stiffness on atherosclerotic plaque amounts. J Biomech Eng 2018;140:05 10071-05100710.

67. Jaminon A, Reesink K, Kroon A, et al. The role of vascular smooth muscle cells in arterial remodeling: focus on calcification-related processes. Int J Mol Sci 2019;20:5694.

68. Mikael LR, Paiva AMG, Gomes MM, et al. Vascular aging and arterial stiffness. Arq Bras Cardiol 2017;109:253-8.

69. Lacolley P, Regnault V, Segers P, et al. Vascular smooth muscle cells and arterial stiffening: relevance in development, aging, and disease. Physiological Reviews 2017;97:1555-617.

70. Currie S, Hoggard N, Craven IJ, et al. Understanding MRI: basic MR physics for physicians. Postgrad Med J 2013;89:209-23.

71. Council NR. Effects of Electromagnetic Fields on Organs and Tissues. Assessment of the Possible Health Effects of Ground Wave Emergency Network. National Academies Press (US); 1993.

72. Salvado O, Hillenbrand C, Suri J, et al., editors. MR coil sensitivity inhomogeneity correction for plaque characterization in carotid arteries. Medical Imaging 2004: Image Processing; 2004: International Society for Optics and Photonics.

73. Kalender WA. X-ray computed tomography. Phys Med Biol 2006;51:R29.

74. Crawford CR, King KF. Computed tomography scanning with simultaneous patient translation. Med Phys 1990;17:967-82.

75. Saba L, di Martino M, Siotto P, et al. Radiation dose and image quality of computed tomography of the supra-aortic arteries: A comparison between single-source and dualsource CT Scanners. J Neuroradiol 2018;45:136-41.

76. Saba L, Argioas GM, Lucatelli P, et al. Variation of degree of stenosis quantification using different energy level with dual energy CT scanner. Neuroradiology 2019;61:285-91.

77. Saba L, Sanfilippo R, di Martino M, et al. Volumetric analysis of carotid plaque components and cerebral microbleeds: a correlative study. J Stroke Cerebrovasc Dis 2017;26:552-8.

78. Saba L, Anzidei M, Piga M, et al. Multi-modal CT scanning in the evaluation of cerebrovascular disease patients. Cardiovasc Diagn Ther 2014;4:245.

79. Baradaran H, Ng CR, Gupta A, et al. Extracranial internal carotid artery calcium volume measurement using computer tomography. Int Angiol 2017;36:445-61.

80. Saba L, Argiolas GM, Raz E, et al. Carotid artery dissection on non-contrast CT: Does color improve the diagnostic confidence? Eur J Radiol 2014;83:2288-93.

81. Hagood NW, McFarland AJ. Modeling of a piezoelectric rotary ultrasonic motor. IEEE Trans Ultrason Ferroelectr Freq Control 1995;42:210-24.

82. Porcu M, Anzidei M, Suri JS, et al. Carotid artery imaging: 
The study of intra-plaque vascularization and hemorrhage in the era of the "vulnerable" plaque. J Neuroradiol 2020;47:464-72.

83. Murgia A, Erta M, Suri JS, et al. CT imaging features of carotid artery plaque vulnerability. Ann Transl Med 2020;8:1261.

84. Saba L, Mannelli L, Balestrieri A, et al. CT and MR Imaging of Carotid Wall and Plaque. Journal of Neurosonology and Neuroimaging 2019;11:115-25.

85. Lairez O, Hyafil F, editors. A Clinical Role of PET in Atherosclerosis and Vulnerable Plaques? Seminars in Nuclear Medicine; 2020: Elsevier.

86. Zhu G, Hom J, Li Y, et al. Carotid plaque imaging and the risk of atherosclerotic cardiovascular disease. Cardiovasc Diagn Ther 2020;10:1048.

87. Hussain MA, Saposnik G, Raju S, et al. Association between statin use and cardiovascular events after carotid artery revascularization. J Am Heart Assoc 2018;7:e009745.

88. Arnold JA, Modaresi KB, Thomas N, et al. Carotid plaque characterization by duplex scanning: observer error may undermine current clinical trials. Stroke 1999;30:61-5.

89. Kakkos SK, Nicolaides AN, Kyriacou E, et al. Computerized texture analysis of carotid plaque ultrasonic images can identify unstable plaques associated with ipsilateral neurological symptoms. Angiology 2011;62:317-28.

90. Liu W, Xie Y, Wang C, et al. Atherosclerosis T1-weighted characterization (CATCH): evaluation of the accuracy for identifying intraplaque hemorrhage with histological validation in carotid and coronary artery specimens. J Cardiovasc Magn Reson 2018;20:27.

91. Sharma AM, Gupta A, Kumar PK, et al. A review on carotid ultrasound atherosclerotic tissue characterization and stroke risk stratification in machine learning framework. Curr Atheroscler Rep 2015;17:55.

92. Acharya UR, Sree SV, Mookiah M, et al. Computed tomography carotid wall plaque characterization using a combination of discrete wavelet transform and texture features: A pilot study. Proc Inst Mech Eng H 2013;227:643-54.

93. Acharya UR, Faust O, Alvin A, et al. Understanding symptomatology of atherosclerotic plaque by imagebased tissue characterization. Comput Methods Programs Biomed 2013;110:66-75.

94. Acharya UR, Molinari F, Saba L, et al., editors. Carotid ultrasound symptomatology using atherosclerotic plaque characterization: a class of Atheromatic systems. 2012 Annual International Conference of the IEEE Engineering in Medicine and Biology Society; 2012: IEEE.

95. Molinari F, Raghavendra U, Gudigar A, et al. An efficient data mining framework for the characterization of symptomatic and asymptomatic carotid plaque using bidimensional empirical mode decomposition technique. Med Biol Eng Comput 2018;56:1579-93.

96. Acharya UR, Krishnan MMR, Sree SV, et al. Plaque tissue characterization and classification in ultrasound carotid scans: a paradigm for vascular feature amalgamation. IEEE Trans Instrum Meas 2012;62:392-400.

97. Christodoulou CI, Kyriacou E, Pattichis MS, et al., editors. A comparative study of morphological and other texture features for the characterization of atherosclerotic carotid plaques. International Conference on Computer Analysis of Images and Patterns; 2003: Springer.

98. Huang X, Zhang Y, Qian M, et al. Classification of carotid plaque echogenicity by combining texture features and morphologic characteristics. J Ultrasound Med 2016;35:2253-61.

99. Kyriacou E, Nicolaides A, Pattichis CS, et al., editors. First and second order statistical texture features in carotid plaque image analysis: Preliminary results from ongoing research. 2011 Annual International Conference of the IEEE Engineering in Medicine and Biology Society; 2011: IEEE.

100. Sanagala SS, Gupta SK, Koppula VK, et al., editors. A Fast and Light Weight Deep Convolution Neural Network Model for Cancer Disease Identification in Human Lung (s). 2019 18th IEEE International Conference On Machine Learning And Applications (ICMLA); 2019: IEEE.

101. Geroulakos G, Ramaswami G, Nicolaides A, et al. Characterization of symptomatic and asymptomatic carotid plaques using high-resolution real-time ultrasonography. Br J Surg 1993;80:1274-7.

102.Kakkos SK, Nicolaides AN, Geroulakos G, et al. Effect of computer monitor brightness on visual (subjective) carotid plaque characterization. J Clin Ultrasound 2011;39:497-501.

103. Boulos NM, Gardin JM, Malik S, et al. Carotid plaque characterization, stenosis, and intima-media thickness according to age and gender in a large registry cohort. Am J Cardiol 2016;117:1185-91.

104. Czernuszewicz TJ, Homeister JW, Caughey MC, et al. Performance of acoustic radiation force impulse ultrasound imaging for carotid plaque characterization with histologic validation. J Vasc Surg 2017;66:1749-57.e3.

105.Demarco JK, Rutt BK, Clarke SE. Carotid plaque characterization by magnetic resonance imaging: review of 
the literature. Top Magn Reson Imaging 2001;12:205-17.

106. Ota H, Tamura H, Itabashi R, et al. Quantitative characterization of carotid plaque components using $M R$ apparent diffusion coefficients and longitudinal relaxation rates at 3T: a comparison with histology. J Magn Reson Imaging 2018;48:1657-67.

107.Liu Y, Luan Q. Association between chronic periodontitis and carotid intima-media thickness of middle-aged and older adults in Shijingshan community of Beijing. Beijing da xue xue bao Yi xue ban 2018;50:264-70.

108. Hatsukami TS, Yuan C. MRI in the early identification and classification of high-risk atherosclerotic carotid plaques. Imaging Med 2010;2:63.

109.von Ingersleben G, Schmiedl U, Hatsukami T, et al. Characterization of atherosclerotic plaques at the carotid bifurcation: correlation of high-resolution MR imaging with histologic analysis--preliminary study. Radiographics 1997;17:1417-23.

110. Chu B, Phan BAP, Balu N, et al. Reproducibility of carotid atherosclerotic lesion type characterization using high resolution multicontrast weighted cardiovascular magnetic resonance. J Cardiovasc Magn Reson 2006;8:793-9.

111.Zhao X, Hippe DS, Li R, et al. Prevalence and characteristics of carotid artery high-risk atherosclerotic plaques in Chinese patients with cerebrovascular symptoms: a Chinese atherosclerosis risk evaluation II study. J Am Heart Assoc 2017;6:e005831.

112. Wintermark M, Glastonbury C, Tong E, et al. Semiautomated computer assessment of the degree of carotid artery stenosis compares favorably to visual evaluation. J Neurol Sci 2008;269:74-9.

113. Chien JD, Furtado A, Cheng SC, et al. Demographics of carotid atherosclerotic plaque features imaged by computed tomography. J Neuroradiol 2013;40:1-10.

114. Wintermark M, Arora S, Tong E, et al. Carotid plaque computed tomography imaging in stroke and nonstroke patients. Ann Neurol 2008;64:149-57.

115.Li Y, Zhu G, Ding V, et al. Assessing the Relationship between Atherosclerotic Cardiovascular Disease Risk Score and Carotid Artery Imaging Findings. J Neuroimaging 2019;29:119-25.

116. Griffin MB, Kyriacou E, Pattichis C, et al. Juxtaluminal hypoechoic area in ultrasonic images of carotid plaques and hemispheric symptoms. J Vasc Surg 2010;52:69-76.

117. Nicolaides A, Griffin M, Makris GC, et al. Carotid Plaque Texture Analysis Using 3-Dimensional Volume Ultrasonic Imaging. Ultrasound and Carotid Bifurcation Atherosclerosis. Springer; 2011. p. 299-324.
118. Loizou CP, Pantziaris M, Theofilou M, et al., editors. Texture Analysis in Ultrasound Images of Carotid Plaque Components of Asymptomatic and Symptomatic Subjects. IFIP International Conference on Artificial Intelligence Applications and Innovations; 2013: Springer.

119.Jamthikar AD, Gupta D, Saba L, et al. Artificial intelligence framework for predictive cardiovascular and stroke risk assessment models: A narrative review of integrated approaches using carotid ultrasound. Comput Biol Med 2020;126:104043.

120. Chiu B, Shamdasani V, Entrekin R, et al. Characterization of carotid plaques on 3-dimensional ultrasound imaging by registration with multicontrast magnetic resonance imaging. J Ultrasound Med 2012;31:1567-80.

121.El-Baz AS, Acharya R, Mirmehdi M, et al. Multi modality state-of-the-art medical image segmentation and registration methodologies. Springer Science \& Business Media; 2011.

122. Guo Y, Sivaramakrishna R, Lu C-C, et al. Breast image registration techniques: a survey. Med Biol Eng Comput 2006;44:15-26.

123. Murata K, Murata N, Chu B, et al. Characterization of carotid atherosclerotic plaques using 3-dimensional MERGE magnetic resonance imaging and correlation with stroke risk factors. Stroke 2020;51:475-80.

124.Guan Q, Du B, Teng Z, et al. Bayes clustering and structural support vector machines for segmentation of carotid artery plaques in multicontrast MRI. Comput Math Methods Med 2012;2012.

125.Zhang Q, Qiao H, Dou J, et al. Plaque components segmentation in carotid artery on simultaneous noncontrast angiography and intraplaque hemorrhage imaging using machine learning. Magn Reson Imaging 2019;60:93-100.

126. Fei B, Suri JS, Wilson DL. Three-Dimensional Volume Registration of Carotid MR Images. Stud Health Technol Inform 2005;113:394-411.

127.Zhu G, Li Y, Ding V, et al. Semiautomated characterization of carotid artery plaque features from computed tomography angiography to predict atherosclerotic cardiovascular disease risk score. J Comput Assist Tomogr 2019;43:452-9.

128. Arora S, Chien J, Cheng S-C, et al. Optimal carotid artery coverage for carotid plaque CT-imaging in predicting ischemic stroke. J Neuroradiol 2010;37:98-103.

129. He C, Li Z, Wang J, et al. Atherosclerotic Plaque Tissue Characterization: An OCT-Based Machine Learning Algorithm With ex vivo Validation. Front Bioeng 
Biotechnol 2020;8:749.

130. Acharya UR, Sree SV, Krishnan MMR, et al. Non-invasive automated 3D thyroid lesion classification in ultrasound: a class of ThyroScan ${ }^{\mathrm{TM}}$ systems. Ultrasonics 2012;52:508-20.

131. Acharya UR, Vinitha Sree S, Mookiah M, et al. Diagnosis of Hashimoto's thyroiditis in ultrasound using tissue characterization and pixel classification. Proc Inst Mech Eng H 2013;227:788-98.

132. Acharya UR, Swapna G, Sree SV, et al. A review on ultrasound-based thyroid cancer tissue characterization and automated classification. Technol Cancer Res Treat 2014;13:289-301.

133. Ribeiro R, Marinho RT, Velosa J, et al., editors. The usefulness of ultrasound in the classification of chronic liver disease. 2011 Annual International Conference of the IEEE Engineering in Medicine and Biology Society; 2011: IEEE.

134. Acharya UR, Molinari F, Sree SV, et al. Ovarian tissue characterization in ultrasound: a review. Technol Cancer Res Treat 2015;14:251-61.

135. Acharya UR, Saba L, Molinari F, et al., editors. Ovarian tumor characterization and classification: A class of GyneScan ${ }^{\mathrm{TM}}$ systems. 2012 Annual International Conference of the IEEE Engineering in Medicine and Biology Society; 2012: IEEE.

136. Acharya UR, Sree SV, Kulshreshtha S, et al. GyneScan: an improved online paradigm for screening of ovarian cancer via tissue characterization. Technol Cancer Res Treat 2014;13:529-39.

137. Acharya UR, Sree SV, Krishnan MMR, et al. Ovarian tumor characterization using 3D ultrasound. Technol Cancer Res Treat 2012;11:543-52.

138. Acharya UR, Sree SV, Molinari F, et al. An automated technique for carotid far wall classification using grayscale features and wall thickness variability. J Clin Ultrasound 2015;43:302-11.

139. Araki T, Ikeda N, Shukla D, et al. PCA-based polling strategy in machine learning framework for coronary artery disease risk assessment in intravascular ultrasound: a link between carotid and coronary grayscale plaque morphology. Comput Methods Programs Biomed 2016;128:137-58.

140. Banchhor SK, Londhe ND, Araki T, et al. Calcium detection, its quantification, and grayscale morphologybased risk stratification using machine learning in multimodality big data coronary and carotid scans: a review. Comput Biol Med 2018;101:184-98.
141.Cuadrado-Godia E, Dwivedi P, Sharma S, et al. Cerebral small vessel disease: A review focusing on pathophysiology, biomarkers, and machine learning strategies. J Stroke 2018;20:302.

142.El-Baz A, Suri JS. Lung imaging and computer aided diagnosis. CRC Press; 2011.

143. Seabra JC, Pedro LM, e Fernandes JF, et al. A 3-D ultrasound-based framework to characterize the echo morphology of carotid plaques. IEEE Trans Biomed Eng 2009;56:1442-53.

144. Seabra J, Pedro LM, e Fernandes JF, et al., editors. Ultrasonographic characterization and identification of symptomatic carotid plaques. 2010 Annual International Conference of the IEEE Engineering in Medicine and Biology; 2010: IEEE.

145.Afonso D, Seabra J, Suri JS, et al., editors. A CAD system for atherosclerotic plaque assessment. 2012 Annual International Conference of the IEEE Engineering in Medicine and Biology Society; 2012: IEEE.

146.Loizou CP, Pattichis CS, Pantziaris M, et al. Texture feature variability in ultrasound video of the atherosclerotic carotid plaque. IEEE J Transl Eng Health Med 2017;5:1800509.

147.Doonan RJ, Dawson A, Kyriacou E, et al. Association of ultrasonic texture and echodensity features between sides in patients with bilateral carotid atherosclerosis. Eur J Vasc Endovasc Surg 2013;46:299-305.

148. Araki T, Ikeda N, Dey N, et al. Shape-based approach for coronary calcium lesion volume measurement on intravascular ultrasound imaging and its association with carotid intima-media thickness. J Ultrasound Med 2015;34:469-82.

149. Kyriacou E, Pattichis MS, Pattichis CS, et al. Classification of atherosclerotic carotid plaques using morphological analysis on ultrasound images. Applied Intelligence 2009;30:3-23.

150. Kyriacou EC, Petroudi S, Pattichis CS, et al. Prediction of high-risk asymptomatic carotid plaques based on ultrasonic image features. IEEE Trans Inf Technol Biomed 2012;16:966-73.

151.Saba L, Jain PK, Suri HS, et al. Plaque tissue morphologybased stroke risk stratification using carotid ultrasound: a polling-based PCA learning paradigm. J Med Syst 2017;41:98.

152. Christodoulou C, Pattichis C, Kyriacou E, et al. Image retrieval and classification of carotid plaque ultrasound images. The Open Cardiovascular Imaging Journal 2010;2. 153. Afonso D, Seabra J, Pedro LM, et al. An ultrasonographic 
risk score for detecting symptomatic carotid atherosclerotic plaques. IEEE J Biomed Health Inform 2015;19:1505-13.

154. Seabra JC, Ciompi F, Pujol O, et al. Rayleigh mixture model for plaque characterization in intravascular ultrasound. IEEE Trans Biomed Eng 2011;58:1314-24.

155.Pattichis C, Christodoulou C, Pattichis M, et al., editors. An integrated system for the assessment of ultrasonic imaging atherosclerotic carotid plaques. Proceedings 2001 International Conference on Image Processing (Cat. No. 01CH37205); 2001: IEEE.

156.Papadopoulos H, Kyriacou E, Nicolaides A, et al., editors. Reliable probabilistic prediction of high-risk asymptomatic carotid plaques. Proceedings of the 16th International Conference on Engineering Applications of Neural Networks (INNS); 2015.

157.Papadopoulos H, Kyriacou E, Nicolaides A. Unbiased confidence measures for stroke risk estimation based on ultrasound carotid image analysis. Neural Computing and Applications 2017;28:1209-23.

158. Mougiakakou SG, Golemati S, Gousias I, et al. Computeraided diagnosis of carotid atherosclerosis based on ultrasound image statistics, laws' texture and neural networks. Ultrasound Med Biol 2007;33:26-36.

159. Roy-Cardinal M-H, Destrempes F, Soulez G, et al. Assessment of carotid artery plaque components with machine learning classification using homodyned-K parametric maps and elastograms. IEEE Trans Ultrason Ferroelectr Freq Control 2019;66:493-504.

160.Le EPV, Evans N, Tarkin J, et al. 105 Machine learning and carotid artery CT radiomics identify significant differences between culprit and non-culprit lesions in patients with stroke and transient ischaemic attack. BMJ Publishing Group Ltd and British Cardiovascular Society; 2020.

161. Meshram NH, Mitchell CC, Wilbrand S, et al. Deep Learning for Carotid Plaque Segmentation using a Dilated U-Net Architecture. Ultrasonic Imaging 2020;42:221-30.

162.Zhou R, Fenster A, Xia Y, et al. Deep learning-based carotid media-adventitia and lumen-intima boundary segmentation from three-dimensional ultrasound images. Med Phys 2019;46:3180-93.

163. Ronneberger O, Fischer P, Brox T, editors. U-net: Convolutional networks for biomedical image segmentation. International Conference on Medical image computing and computer-assisted intervention; 2015: Springer.

164. Wu X, Zhao Y, Radev D, et al. Identification of patients with carotid stenosis using natural language processing.
Eur Radiol 2020;30:4125-33.

165. Ryoo HG, Choi H, Lee DS. Deep learning-based interpretation of basal/acetazolamide brain perfusion SPECT leveraging unstructured reading reports. Eur J Nucl Med Mol Imaging 2020;47:2186-96.

166. Groves LA, VanBerlo B, Veinberg N, et al. Automatic segmentation of the carotid artery and internal jugular vein from 2D ultrasound images for 3D vascular reconstruction. Int J Comput Assist Radiol Surg 2020;15:1835-46.

167.Demirer M, Candemir S, Bigelow MT, et al. A User Interface for Optimizing Radiologist Engagement in Image Data Curation for Artificial Intelligence. Radiol Artif Intell 2019;1:e180095.

168. Lee J, Prabhu D, Kolluru C, et al. Fully automated plaque characterization in intravascular OCT images using hybrid convolutional and lumen morphology features. Sci Rep 2020;10:2596.

169.Lekadir K, Galimzianova A, Betriu À, et al. A convolutional neural network for automatic characterization of plaque composition in carotid ultrasound. IEEE J Biomed Health Inform 2017;21:48-55.

170. Saba L, Sanagala SS, Gupta SK, et al. Ultrasound-based internal carotid artery plaque characterization using deep learning paradigm on a supercomputer: a cardiovascular disease/stroke risk assessment system. Int J Cardiovasc Imaging 2021;37:1511-28.

171. Mansoor A, Perez G, Nino G, et al., editors. Automatic tissue characterization of air trapping in chest radiographs using deep neural networks. 2016 38th Annual International Conference of the IEEE Engineering in Medicine and Biology Society (EMBC); 2016: IEEE.

172.Abdolmanafi A, Duong L, Dahdah N, et al. Characterization of coronary artery pathological formations from OCT imaging using deep learning. Biomedical Optics Express 2018;9:4936-60.

173. Savaş S, Topaloğlu N, Kazcı Ö, et al. Classification of Carotid Artery Intima Media Thickness Ultrasound Images with Deep Learning. J Med Syst 2019;43:273.

174. Biswas M, Saba L, Chakrabartty S, et al. Two-stage artificial intelligence model for jointly measurement of atherosclerotic wall thickness and plaque burden in carotid ultrasound: A screening tool for cardiovascular/stroke risk assessment. Comput Biol Med 2020;123:103847.

175.Jamthikar AD, Khanna NN, Piga M, et al. Rheumatoid arthritis: its link to atherosclerosis imaging and cardiovascular risk assessment using machine-learningbased tissue characterization. Vascular and Intravascular Imaging Trends, Analysis, and Challenges, Volume 2. IOP 
Publishing; 2019. p. 4-1-4-29.

176. Rasmussen D, Voelker A, Eliasmith C. A neural model of hierarchical reinforcement learning. PLoS One 2017;12:e0180234.

177.Zhao X, Underhill HR, Zhao Q, et al. Discriminating carotid atherosclerotic lesion severity by luminal stenosis and plaque burden: a comparison utilizing highresolution magnetic resonance imaging at 3.0 Tesla. Stroke 2011;42:347-53.

178. Gao S, van 't Klooster R, Kitslaar PH, et al. Learning based automated segmentation of the carotid artery vessel wall in dual-sequence MRI using subdivision surface fitting. Med Phys 2017;44:5244-59.

179. Li B, Jiao Y, Fu C, et al. Contralateral artery enlargement predicts carotid plaque progression based on machine learning algorithm models in apoE-/- mice. Biomed Eng Online 2016;15:146.

180. Christodoulou CI, Kyriacou E, Pattichis MS, et al. Plaque feature extraction. Ultrasound and carotid bifurcation atherosclerosis. Springer; 2011. p. 223-45.

181. Bizopoulos PA, Sakellarios AI, Koutsouris DD, et al., editors. Prediction of atheromatic plaque evolution in carotids using features extracted from the arterial geometry. 2015 37th Annual International Conference of the IEEE Engineering in Medicine and Biology Society (EMBC); 2015: IEEE.

182. Colak C, Karaman E, Turtay MG. Application of knowledge discovery process on the prediction of stroke. Comput Methods Programs Biomed 2015;119:181-5.

183. Qian C, Yang X. An integrated method for atherosclerotic carotid plaque segmentation in ultrasound image. Comput Methods Programs Biomed 2018;153:19-32.

184.Zhou R, Luo Y, Fenster A, et al. Fractal dimension based carotid plaque characterization from three-dimensional ultrasound images. Med Biol Eng Comput 2019;57:135-46.

185. Viswanathan V, Jamthikar A, Gupta D, et al. Low-cost preventive screening using carotid ultrasound in patients with diabetes. Front Biosci (Landmark Ed) 2020;25:1132.

186. Tsakanikas VD, Siogkas PK, Mantzaris MD, et al., editors. A deep learning oriented method for automated 3D reconstruction of carotid arterial trees from MR imaging. 2020 42nd Annual International Conference of the IEEE Engineering in Medicine \& Biology Society (EMBC); 2020: IEEE.

187. Wu J, Xin J, Yang X, et al. Deep morphology aided diagnosis network for segmentation of carotid artery vessel wall and diagnosis of carotid atherosclerosis on black blood vessel wall MRI. Med Phys 2019;46:5544-61.

188. Sheth SA, Lopez-Rivera V, Barman A, et al. Machine Learning-Enabled Automated Determination of Acute Ischemic Core From Computed Tomography Angiography. Stroke 2019;50:3093-100.

189.van Engelen A, Van Dijk AC, Truijman MT, et al. Multicenter MRI carotid plaque component segmentation using feature normalization and transfer learning. IEEE Trans Med Imaging 2015;34:1294-305.

190. Yahagi K, Kolodgie FD, Lutter C, et al. Pathology of human coronary and carotid artery atherosclerosis and vascular calcification in diabetes mellitus. Arterioscler Thromb Vasc Biol 2017;37:191-204.

191. Sigala F, Oikonomou E, Antonopoulos AS, et al. Coronary versus carotid artery plaques. Similarities and differences regarding biomarkers morphology and prognosis. Curr Opin Pharmacol 2018;39:9-18.

192. Fabiano S, Mancino S, Stefanini M, et al. High-resolution multicontrast-weighted MR imaging from human carotid endarterectomy specimens to assess carotid plaque components. Eur Radiol 2008;18:2912-21.

193. Virmani R, Burke AP, Farb A, et al. Pathology of the vulnerable plaque. J Am Coll Cardiol 2006;47:C13-8.

194. Virmani R, Burke AP, Kolodgie FD, et al. Pathology of the Thin-Cap Fibroatheroma. J Interv Cardiol 2003;16:267-72.

195. Virmani R, Kolodgie FD, Burke AP, et al. Lessons from sudden coronary death: a comprehensive morphological classification scheme for atherosclerotic lesions. Arterioscler Thromb Vasc Biol 2000;20:1262-75.

196. Amato M, Montorsi P, Ravani A, et al. Carotid intimamedia thickness by B-mode ultrasound as surrogate of coronary atherosclerosis: correlation with quantitative coronary angiography and coronary intravascular ultrasound findings. Eur Heart J 2007;28:2094-101.

197. Mosleh W, Adib K, Natdanai P, et al. High-risk carotid plaques identified by CT-angiogram can predict acute myocardial infarction. Int J Cardiovasc Imaging 2017;33:561-8.

198. Jashari F, Ibrahimi P, Nicoll R, et al. Coronary and carotid atherosclerosis: similarities and differences. Atherosclerosis 2013;227:193-200.

199. Bots ML, Grobbee DE. Intima media thickness as a surrogate marker for generalised atherosclerosis. Cardiovasc Drugs Ther 2002;16:341-51.

200.Johri AM, Behl P, Hétu MF, et al. Carotid ultrasound maximum plaque height-a sensitive imaging biomarker for the assessment of significant coronary artery disease. 
Echocardiography 2016;33:281-9.

201.Johri AM, Chitty DW, Matangi M, et al. Can carotid bulb plaque assessment rule out significant coronary artery disease? A comparison of plaque quantification by twoand three-dimensional ultrasound. J Am Soc Echocardiogr 2013;26:86-95.

202. Hamada S, Kashiwazaki D, Yamamoto S, et al. Impact of plaque composition on risk of coronary artery diseases in patients with carotid artery stenosis. J Stroke Cerebrovasc Dis 2018;27:3599-604.

203. Ogata T, Yasaka M, Yamagishi M, et al. Atherosclerosis found on carotid ultrasonography is associated with atherosclerosis on coronary intravascular ultrasonography. J Ultrasound Med 2005;24:469-74.

204.Ishizu T, Ishimitsu T, Kamiya H, et al. The correlation of irregularities in carotid arterial intima-media thickness with coronary artery disease. Heart Vessels 2002;17:1-6.

205.Kablak-Ziembicka A, Tracz W, Przewlocki T, et al. Association of increased carotid intima-media thickness with the extent of coronary artery disease. Heart 2004;90:1286-90.

206. Mantella LE, Colledanchise KN, Hétu M-F, et al. Carotid intraplaque neovascularization predicts coronary artery disease and cardiovascular events. Eur Heart J Cardiovasc Imaging 2019;20:1239-47.

207.Laclaustra M, Casasnovas JA, Fernández-Ortiz A, et al. Femoral and carotid subclinical atherosclerosis association with risk factors and coronary calcium: the AWHS study. J Am Coll Cardiol 2016;67:1263-74.

208. Araki T, Ikeda N, Shukla D, et al. A new method for IVUS-based coronary artery disease risk stratification: a link between coronary \& carotid ultrasound plaque burdens. Comput Methods Programs Biomed 2016;124:161-79.

209.Ikeda N, Gupta A, Dey N, et al. Improved correlation between carotid and coronary atherosclerosis SYNTAX score using automated ultrasound carotid bulb plaque IMT measurement. Ultrasound Med Biol 2015;41:1247-62.

210.Illuminati G, Schneider F, Greco C, et al. Long-term results of a randomized controlled trial analyzing the role of systematic pre-operative coronary angiography before elective carotid endarterectomy in patients with asymptomatic coronary artery disease. Eur J Vasc Endovasc Surg 2015;49:366-74.

211.Gepner AD, Young R, Delaney JA, et al. Comparison of Carotid Plaque Score and Coronary Artery Calcium Score for Predicting Cardiovascular Disease Events: The
Multi-Ethnic Study of Atherosclerosis. J Am Heart Assoc 2017;6:e005179.

212.Araki T, Jain PK, Suri HS, et al. Stroke risk stratification and its validation using ultrasonic Echolucent Carotid Wall plaque morphology: a machine learning paradigm. Comput Biol Med 2017;80:77-96.

213.Zreik M, Van Hamersvelt RW, Wolterink JM, et al. A recurrent $\mathrm{CNN}$ for automatic detection and classification of coronary artery plaque and stenosis in coronary CT angiography. IEEE Trans Med Imaging 2019;38:1588-98.

214. Banchhor SK, Londhe ND, Araki T, et al. Wall-based measurement features provides an improved IVUS coronary artery risk assessment when fused with plaque texture-based features during machine learning paradigm. Comput Biol Med 2017;91:198-212.

215.Komorovsky R, Desideri A. Carotid ultrasound assessment of patients with coronary artery disease: a useful index for risk stratification. Vasc Health Risk Manag 2005;1:131.

216. Acharya UR, Sree SV, Krishnan MMR, et al. Automated classification of patients with coronary artery disease using grayscale features from left ventricle echocardiographic images. Comput Methods Programs Biomed 2013;112:624-32.

217.Jamthikar AD, Gupta D, Mantella LE, et al. Multiclass machine learning vs. conventional calculators for stroke/ CVD risk assessment using carotid plaque predictors with coronary angiography scores as gold standard: a 500 participants study. Int J Cardiovasc Imaging 2021;37:1171-87.

218. Saba L, Atzeni M, Ribuffo D, et al. Analysis of deep inferior epigastric perforator (DIEP) arteries by using MDCTA: comparison between 2 post-processing techniques. Eur J Radiol 2012;81:1828-33.

219. Saba L, Sanfilippo R, Balestrieri A, et al. Relationship between carotid computed tomography dual-energy and brain leukoaraiosis. J Stroke Cerebrovasc Dis 2017;26:1824-30.

220. Beach KW. Principles of Ultrasonic Imaging and Instrumentation. In: Nicolaides A, Beach KW, Kyriacou E et al., editors. Ultrasound and Carotid Bifurcation Atherosclerosis. London: Springer London; 2012. p. 67-96.

221. Nicolaides AN, Kakkos SK, Kyriacou E, et al. Asymptomatic internal carotid artery stenosis and cerebrovascular risk stratification. J Vasc Surg 2010;52:1486-96.e5.

222.Kakkos SK, Griffin MB, Nicolaides AN, et al. The size of juxtaluminal hypoechoic area in ultrasound images of 
asymptomatic carotid plaques predicts the occurrence of stroke. J Vasc Surg 2013;57:609-18.e1.

223. Girshick R, Donahue J, Darrell T, et al., editors. Rich feature hierarchies for accurate object detection and semantic segmentation. Proceedings of the IEEE conference on computer vision and pattern recognition; 2014.

224. Narumi S, Sasaki M, Natori T, et al. Carotid plaque characterization using 3D T1-weighted MR imaging with histopathologic validation: a comparison with $2 \mathrm{D}$ technique. AJNR Am J Neuroradiol 2015;36:751-6.

225.Sakellarios AI, Stefanou K, Siogkas P, et al. Novel methodology for 3D reconstruction of carotid arteries and plaque characterization based upon magnetic resonance imaging carotid angiography data. Magn Reson Imaging 2012;30:1068-82.

Cite this article as: Saba L, Sanagala SS, Gupta SK, Koppula VK, Johri AM, Khanna NN, Mavrogeni S, Laird JR, Pareek G, Miner M, Sfikakis PP, Protogerou A, Misra DP, Agarwal V, Sharma AM, Viswanathan V, Rathore VS, Turk M, Kolluri R, Viskovic K, Cuadrado-Godia E, Kitas GD, Sharma N, Nicolaides A, Suri JS. Multimodality carotid plaque tissue characterization and classification in the artificial intelligence paradigm: a narrative review for stroke application. Ann Transl Med 2021;9(14):1206. doi: 10.21037/atm-20-7676
226.Perrotta P, Pintelon I, de Vries MR, et al. ThreeDimensional Imaging of Intraplaque Neovascularization in a Mouse Model of Advanced Atherosclerosis. J Vasc Res 2020;57:348-54.

227. Murgia A, Balestrieri A, Francone M, et al. Plaque imaging volume analysis: technique and application. Cardiovasc Diagn Ther 2020;10:1032-47.

228. Saba L, Raz E, di Martino M, et al. Is there an association between asymmetry of carotid artery wall thickness (ACAWT) and cerebrovascular symptoms? Int J Neurosci 2015;125:456-63.

229. Tandel GS, Balestrieri A, Jujaray T, et al. Multiclass magnetic resonance imaging brain tumor classification using artificial intelligence paradigm. Comput Biol Med 2020;122:103804. 